\title{
STOCHASTIC PROCESSES ON TOTALLY DISCONNECTED TOPOLOGICAL GROUPS
}

\author{
S. V. LUDKOVSKY
}

Received 27 August 2001

\begin{abstract}
Stochastic processes on totally disconnected topological groups are investigated. In particular, they are considered for diffeomorphism groups and loop groups of manifolds on non-Archimedean Banach spaces. Theorems about a quasi-invariance and a pseudodifferentiability of transition measures are proved. Transition measures are used for the construction of strongly continuous representations including the irreducible ones of these groups. In addition, stochastic processes on general Banach-Lie groups, loop monoids, loop spaces, and path spaces of manifolds on Banach spaces over non-Archimedean local fields are also investigated.
\end{abstract}

2000 Mathematics Subject Classification: 28C20, 46 S10.

1. Introduction. This paper is a continuation of [20, 21], where stochastic processes on Banach spaces over local fields and stochastic antiderivational equations on them were investigated. This paper is devoted to stochastic processes on a totally disconnected topological group which is complete, separable, and ultrametrizable. In particular, stochastic processes on diffeomorphism groups and loop groups of manifolds on Banach spaces over a local field are considered. These groups were defined and investigated in [13, 15, 16, 18]. These groups are nonlocally compact and for them the Campbell-Hausdorff formula is not valid (in an open local subgroup). In this paper, topological groups locally satisfying the Campbell-Hausdorff formula are also considered.

Finite-dimensional Lie groups locally satisfy the Campbell-Hausdorff formula. This is guaranteed, if to impose two conditions on a locally compact topological Hausdorff group $G$ : it is a $C^{\infty}$-manifold and the following mapping $(f, g) \mapsto f \circ g^{-1}$ from $G \times G$ into $G$ is of class $C^{\infty}$. But for infinite-dimensional $G$, the Campbell-Hausdorff formula does not follow from these conditions. Frequently, topological Hausdorff groups satisfying these two conditions also are called Lie groups, though they cannot have all properties of finite-dimensional Lie groups, so that, the Lie algebras for them do not play the same role as in the finite-dimensional case and therefore Lie algebras are not so helpful. If $G$ is a Lie group and its tangent space $T_{e} G$ is a Banach space, then it is called a Banach-Lie group, sometimes it is undermined, that they satisfy the CampbellHausdorff formula locally for a Banach-Lie algebra $T_{e} G$. In some papers, the Lie group terminology undermines, that it is finite-dimensional. It is worthwhile to call Lie groups satisfying the Campbell-Hausdorff formula locally (in an open 
local subgroup) by Lie groups in the narrow sense; in the contrary case, to call them by Lie groups in the broad sense.

In this paper, also theorems about a quasi-invariance and a pseudodifferentiability of transition measures on the totally disconnected topological group $G$ relative to the dense subgroup $G^{\prime}$ are proved. In each concrete case of $G$, it is necessary to construct a stochastic process and $G^{\prime}$. Below, path spaces, loop spaces, loop monoids, loop groups, and diffeomorphism groups are considered not only for finite-dimensional, but also for infinite-dimensional manifolds.

In particular, loop and diffeomorphism groups are important for the development of the representation theory of nonlocally compact groups. Their representation theory has many differences with the traditional representation theory of locally compact groups and finite-dimensional Lie groups, because nonlocally compact groups have not $C^{*}$-algebras associated with the Haar measures and they have not underlying Lie algebras and relations between representations of groups and underlying algebras (see also [17]).

In view of the A. Weil theorem, if a topological Hausdorff group $G$ has a quasiinvariant measure relative to the entire $G$, then $G$ is locally compact. Since loop groups $\left(L^{M} N\right) \xi$ are not locally compact, they cannot have quasi-invariant measures relative to the entire group, but only relative to proper subgroups $G^{\prime}$ which can be chosen dense in $\left(L^{M} N\right)_{\xi}$, where an index $\xi$ indicates a class of smoothness. The same is true for diffeomorphism groups.

It is necessary to note that there are quite another groups with the same name loop groups, but they are infinite-dimensional Banach-Lie groups of mappings $f: M \rightarrow H$ into a finite-dimensional Lie group $H$ with the pointwise group multiplication of mappings with values in $H$. The loop groups considered here are geometric loop groups.

On the other hand, representation theory of nonlocally compact groups is little developed apart from the case of locally compact groups. For locally compact groups, theory of induced representations is well developed due to works of Frobenius, Mackey, and so forth. But for nonlocally compact groups, it is very little known. In particular, geometric loop and diffeomorphism groups have important applications in modern physical theories (see [16, 18]).

Then, measures are used for the study of associated unitary representations of dense subgroups $G^{\prime}$.

In this paper, notations and definitions from $[18,20,21]$ are used.

\section{Stochastic antiderivational equations and measures on totally disconnected topological groups}

Note 2.1. Let $X$ be a Banach space over a local field $\mathbf{K}$. Suppose $M$ is an analytic manifold modelled on $X$ with an atlas $\operatorname{At}(M)$ consisting of disjoint clopen charts $\left(U_{j}, \phi_{j}\right), j \in \Lambda_{M}, \Lambda_{M} \subset \mathbb{N}$. That is, $U_{j}$ and $\phi_{j}\left(U_{j}\right)$ are clopen in $M$ and $X$, respectively, $\phi_{j}: U_{j} \rightarrow \phi_{j}\left(U_{j}\right)$ are homeomorphisms, and $\phi_{j}\left(U_{j}\right)$ are bounded in $X$. 
Note 2.2. Let $\Omega_{\xi}^{\{k\}}(M, N)$ be the loop submonoid as in [18, Section I.3.5] such that $c>0$ and $c^{\prime}>0$. Then, it generates the loop group $G^{\prime}:=L_{\xi}^{\{k\}}(M, N)$ as in [18, Section II.2.1] such that $G^{\prime}$ is the dense subgroup in $G=L_{\xi}(M, N)$.

REMARK 2.3. Let $M$ be a manifold on the Banach space $X$ with an atlas At $(M)$ consisting of disjunctive charts $\left(U_{j}, \phi_{j}\right), j \in \Lambda, \Lambda \subset \mathbb{N}$, where $U_{j}$ and $\phi_{j}\left(U_{j}\right)$ are clopen in $M$ and $X$, respectively, $\phi_{j}: U_{j} \rightarrow \phi_{j}\left(U_{j}\right)$ is a homeomorphism, also $\phi_{j}\left(U_{j}\right)=B\left(X, x_{j}, r_{j}\right)$ is a ball in $X$ with a radius $0<r_{j}<\infty$ for each $j$ (see also [18, Sections I.2.1-8]).

For $\Lambda=\omega_{0}$, we define a Banach space

$$
\begin{aligned}
\tilde{C}_{*}(t, M \longrightarrow X):= & \left\{\left.f\right|_{U_{j}} \in C_{*}\left(t, U_{j} \longrightarrow X\right),\right. \\
& \|f\|_{C_{*}(t, M \rightarrow X)}:=\sup _{j \in \Lambda}\left(\left\|\left.f\right|_{U_{j}}\right\|_{C_{*}\left(t, U_{j} \rightarrow X\right)} / \min \left(1, r_{j}\right)\right)<\infty, \\
& \left.\left(\left\|\left.f\right|_{U_{j}}\right\|_{C_{*}\left(t, U_{j} \rightarrow X\right)} / \min \left(1, r_{j}\right)\right) \longrightarrow 0 \text { while } j \longrightarrow \infty\right\},
\end{aligned}
$$

where $0 \leq t<\infty, *=0$ for spaces $C_{0}(t, U \rightarrow X), *=\varnothing$ or simply is omitted for $C(t, U \rightarrow X)$. For the finite atlas $\operatorname{At}(M)$, the spaces $\tilde{C}_{*}(t, U \rightarrow X)$ and $C_{*}(t, U \rightarrow$ $X)$ are linearly topologically isomorphic. By $C_{*}^{\theta}(t, M \rightarrow M)$, for $0 \leq t \leq \infty$ is denoted the following space of functions $f: M \rightarrow M$ such that $\left(f_{i}-\theta_{i}\right) \in$ $C_{*}(t, M \rightarrow X)$ for each $i \in \Lambda$ and $f_{i}=\psi_{i} \circ f, \theta_{i}=\psi_{i} \circ \theta$. We introduce the following group:

$$
G(t, M):=\tilde{C}_{0}^{\mathrm{id}}(t, M \longrightarrow M) \cap \operatorname{Hom}(M),
$$

which is called the diffeomorphism group (and the homeomorphism group for $0 \leq t<1$ ), where $\operatorname{Hom}(M)$ is the group of continuous homeomorphisms.

Each function $f \in C_{0}(t, M \rightarrow X)$ has the following decomposition:

$$
\left.f(x)\right|_{U_{j}}=\left.\sum_{\left(i \in \mathbb{N}, n \in \mathbb{N}_{0}\right)} f^{i}(n ; x)\right|_{U_{j}} e_{i} \tilde{z}(n)
$$

and

$$
\left\{e_{i} \tilde{z}(n)\left(\left.\bar{Q}_{m}(x)\right|_{U_{j}}\right): i, n, \operatorname{Ord}(m)=n, j\right\}
$$

is the orthogonal basis, moreover,

$$
\left.f_{n}(x)\right|_{U_{j}}:=\left.\sum_{i} f^{i}(n ; x)\right|_{U_{j}} e_{i} \in C_{0}\left(t, U_{j} \longrightarrow X\right),
$$


where

$$
X_{\tilde{z}(n)}:=\left\{f_{n}(x):\left.f_{n}\right|_{U_{j}} \in C_{0}\left(t, U_{j} \longrightarrow X\right)\right\}
$$

is the Banach space with the norm induced from $C_{0}(t, M \rightarrow X)$ such that

$$
\left.f^{i}(n ; x)\right|_{U_{j}}:=\left.\sum_{\left(\operatorname{Ord} m=n, m=(m(1), \ldots, m(n)), m(j) \in \mathbb{N}_{0}\right)} a\left(m,\left.f^{i}\right|_{U_{j}}\right) \bar{Q}_{m}(x)\right|_{U_{j}},
$$

where $\left.\bar{Q}_{m}(x)\right|_{U_{j}}=0$ for $x \in M \backslash U_{j}$.

For the manifold $M$, we fix a subsequence $\left\{M_{n}: n \in \mathbb{N}_{0}\right\}$ of submanifolds in $M$ such that $M_{n} \hookrightarrow M_{n+1} \hookrightarrow \cdots \hookrightarrow M$ for each $n$, $\operatorname{dim}_{\mathbf{K}} M_{n}=\beta(n) \in \mathbb{N}$ for each $n \in \mathbb{N}_{0}, \bigcup_{n} M_{n}$ is dense in $M$, where $\beta(n)<\beta(n+1)$ for each $n$ and there exists $n_{0} \in \mathbb{N}$ with $\beta(n)=n$ for each $n>n_{0}, \mathbb{N}_{0}:=\mathbb{N} \cup\{0\}$.

We take the following subgroup:

$$
\begin{gathered}
G^{\prime}:=\left\{f \in G(t, M):\left(f^{i}(n ; x)-\mathrm{id}^{i}(n ; x)\right)=: g^{i}(n ; x) \in C_{0}\left(t_{n}, M_{n} \longrightarrow \mathbf{K}\right),\right. \\
\left.\left|a\left(m ;\left.g^{i}(n ; x)\right|_{U_{j}}\right)\right| J_{j}\left(t_{n}, m\right) \leq c(f) p^{v^{\prime}(m, j, i)}\right\},
\end{gathered}
$$

where $c(f)>0$ is a constant, $v^{\prime}(m, j, i)=-c^{\prime} i-c^{\prime} n-c^{\prime \prime} j, n=\operatorname{Ord}(m), c^{\prime}=$ const $>0$ and $c^{\prime \prime}=$ const $\geq 0, c^{\prime \prime}>0$ for $\Lambda=\omega_{0}, t_{n}=t+s(n)$ for $0 \leq t<\infty$, $s(n)>n$ for each $n$, and $\liminf _{n \rightarrow \infty} s(n) / n=: \zeta>1$. Then, there exists the following ultrametric in $G^{\prime}$ :

$$
d(f, \mathrm{id})=\sup _{m, n, j}\left\{\left|a\left(m ;\left.g^{i}(n ; x)\right|_{U_{j}}\right)\right| J_{j}\left(t_{n}, m\right) p^{-v^{\prime}(m, j, i)}\right\} .
$$

Note 2.4. At first it is necessary to prove theorems about the quasiinvariance and the pseudodifferentiability of transition measures of stochastic processes on Banach spaces over local fields. We consider two types of measures on $c_{0}\left(\omega_{0}, \mathbf{K}\right)$. The first is the $q$-Gaussian measure

$$
\mu=\mu_{J, \gamma, q}:=\bigotimes_{j=1}^{\infty} \mu_{j}\left(d x^{j}\right),
$$

where

$$
\mu_{j}\left(d x^{j}\right)=C_{\left|\zeta_{j}\right|-q, \gamma_{j}, q} f_{\left|\zeta_{j}\right|^{-q, \gamma_{j}, q}} v\left(d x^{j}\right)
$$

(see [20, Section 2]). The characteristic functional of the $q$-Gaussian measure is positive definite, hence $\mu$ is nonnegative (see also [11, Section 2.6]). The second is specified below and it is the particular case of measures considered in [21, Theorem 4.2].

Let $w$ be the real-valued nonnegative Haar measure on $\mathbf{K}$ with $w(B(\mathbf{K}, 0,1))=$ 1. We consider the following measure $\mu$ on $c_{0}\left(\omega_{0}, \mathbf{K}\right)$ :

(i) $\mu(d x)=\bigotimes_{j=1}^{\infty} \mu_{j}\left(d x^{j}\right)$, where $x \in c_{0}\left(\omega_{0}, \mathbf{K}\right), x=\left(x^{j}: j \in \omega_{0}\right), x^{j} \in \mathbf{K}$, $x=\sum_{j} x^{j} e_{j}, e_{j}$ is the standard orthonormal base in $c_{0}\left(\omega_{0}, \mathbf{K}\right)$. 
Now, on the Banach space $c_{0}:=c_{0}\left(\omega_{0}, \mathbf{K}\right)$ there is an operator $J \in L_{1}\left(c_{0}\right)$ such that $J e_{i}=v_{i} e_{i}$ with $v_{i} \neq 0$ for each $i$. We consider a measure $v_{i}(d x):=$ $f_{i}(x) w(d x)$ on $\mathbf{K}$, where $f_{i}: \mathbf{K} \rightarrow[0,1]$ is a function belonging to the space $L^{1}(\mathbf{K}, w, \mathbb{R})$ such that $f_{i}(x)=f\left(x / v_{i}\right)+h_{i}\left(x / v_{i}\right)$, where $f$ is a locally constant positive function, $f(x)=\sum_{j=1}^{\infty} C_{j} \mathrm{Ch}_{B_{j}}(x), B_{j}:=B\left(\mathbf{K}, x_{j}, r_{j}\right)$ is a ball in $\mathbf{K}, \mathrm{Ch}_{V}$ is the characteristic function of a subset $V$ in $\mathbf{K}$, that is, $\mathrm{Ch}_{V}(x)=1$ for each $x \in V, \operatorname{Ch}_{V}(x)=0$ for each $x \in \mathbf{K} \backslash V, x_{1}:=0, r_{1}:=1, \inf _{j} r_{j}=1,\left\{B_{j}: j\right\}$ is the disjoint covering of $\mathbf{K}, 1 \geq C_{j}>0$, $\lim _{|x| \rightarrow \infty} f(x)=0, h_{i} \in L^{1}(\mathbf{K}, w, \mathbb{R})$ such that $\operatorname{ess}_{w}-\sup _{x \in \mathbf{K}}\left|h_{i}(x) / f(x)\right|=\delta_{i}<1, \sum_{i} \delta_{i}<\infty$, and $v_{i}(\mathbf{K})=1$. Then, $v_{i}(S)>0$ for each open subset $S$ in $\mathbf{K}$. There exists a $\sigma$-additive product measure;

(ii) $\mu_{J}(d x):=\prod_{i=1}^{\infty} \mu_{i}\left(d x^{i}\right)$ on the $\sigma$-algebra of Borel subsets of $c_{0}$ since the Borel $\sigma$-algebras defined for the weak topology of $c_{0}$ and for the norm topology of $c_{0}$ coincide, where $\mu_{i}\left(d x^{i}\right):=v\left(d x^{i} / v_{i}\right)$.

Let $A: c_{0} \rightarrow c_{0}$ be a linear topological isomorphism, that is, $A, A^{-1} \in L\left(c_{0}\right)$, then for a measure $\mu$ on $c_{0}$ there exists its image $\mu_{A}(S):=\mu\left(A^{-1} S\right)$ for each Borel subset $S$ in $c_{0}$. In view of [21, Proposition 2.10] $L_{q}\left(c_{0}\right)$ is the ideal in $L\left(c_{0}\right)$. This produces new $q$-Gaussian measures $\left(\mu_{J, \gamma, q}\right)_{A}=: \mu_{A J, A^{*} \gamma, q}$ and measures of the second type $\left(\mu_{J}\right)_{A}=: \mu_{A J}$. In view of [21, Remark 2.5], each injective linear operator $S \in L_{q}\left(c_{0}\right)$ with $S\left(c_{0}\right)$ dense in $c_{0}$ can be presented in the form $S=A J$. Hence, for each such $S$ there exist the $\sigma$-additive measures $\mu_{S, S * \gamma, q}$ and $\mu_{S}$. These measures are induced by the corresponding cylinder measures $\mu_{I, \gamma, q}$ or $\mu_{I}$ on $\mathbf{K}^{\aleph_{0}}$, where $I$ is the unit operator, since $c_{0}$ in the weak topology is isomorphic with $\mathbf{K}^{\mathrm{N}_{0}}$. Here, the algebra $\boldsymbol{U}$ of cylindrical subsets is generated by subsets $\pi_{V}^{-1}(A)$, where $A$ is a Borel subset in $\mathbf{K}^{n}, \operatorname{card}(V)=n<\kappa_{0}, V \subset \mathbb{N}$, $\pi_{V}: \mathbf{K}^{\aleph_{0}} \rightarrow \prod_{i \in V} \mathbf{K}_{i}$ is the natural projection.

On the space $C_{0}^{0}(T, H)=C_{0}^{0}(T, \mathbf{K}) \otimes H$, let $S=S_{1} \otimes S_{2}$ and $\gamma=\gamma^{1} \otimes \gamma^{2}$, where $S_{1}$ is a linear operator on $C_{0}^{0}(T, \mathbf{K})$ and $S_{2}$ is a linear operator on $H, \gamma^{1} \in$ $C_{0}^{0}(T, \mathbf{K}), \gamma^{2} \in H$ such that the measure $\mu_{S, \gamma, q}$ is the product of measures $\mu_{S_{1}, \gamma^{1}, q}$ on $C_{0}^{0}(T, \mathbf{K})$ and $\mu_{S_{2}, \gamma^{2}, q}$ on $H$, analogously $\mu_{S}$ is the product of measures $\mu_{S_{1}}$ on $C_{0}^{0}(T, \mathbf{K})$ and $\mu_{S_{2}}$ on $H$. With the help of such measures on the space $C_{0}^{0}(T, H)$, the stochastic process $w(t, \omega)$ is defined as in [21, Definition 4.1 and Theorem 4.2] and [20, Sections 3.1 and 3.2].

2.1. Let $Y$ be a Banach space over the local field $\mathbf{K}$ and $V$ a neighbourhood of zero in $Y$. Consider either the measure $\mu_{S, \gamma, \psi}$ or $\mu_{S}$ outlined in Note 2.4. Suppose that in stochastic antiderivational equations [20, (3.8) and Theorem 3.4(i)], mappings $a$ and $E$ are dependent on the parameter $y \in V$, that is, $a=a(t, \omega, \xi, y)$ and $E=E(t, \omega, \xi, y)$; moreover, $a_{k, l}=a_{k, l}(t, \xi, y)$ for each $k$ and $l$ in the latter equation, [20, condition (LLC)] is satisfied for each $0<r<\infty$ with the constant $K_{r}$ independent from $y \in V$ for each $y \in V$. Evidently, $[20,(3.8)]$ is the particular case of [20, Theorem 3.4(i)], when in the latter equation the corresponding $a_{0,1}$ and $a_{1,0}$ are chosen with all the others $a_{k, l}=0$ (when $k+l \neq 1$ ). Also let 
(i) $a, E$, and $a_{k, l}$ be of class $C^{1}$ by $y \in V$ such that

$$
\begin{aligned}
a & \in C^{1}\left(V, L^{q}\left(\Omega, \mathscr{F}, \lambda ; C^{0}\left(B_{R}, L^{q}\left(\Omega, \mathscr{F}, \lambda ; C^{0}\left(B_{R}, H\right)\right)\right)\right)\right), \\
E & \in C^{1}\left(V, L^{r}\left(\Omega, \mathscr{F}, \lambda ; C^{0}\left(B_{R}, L\left(L^{q}\left(\Omega, \mathscr{F}, \lambda ; C^{0}\left(B_{R}, H\right)\right)\right)\right)\right),\right. \\
a_{m-l, l} & \in C^{1}\left(V, C^{0}\left(B_{R_{1}} \times B\left(L^{q}\left(\Omega, \mathscr{F}, \lambda ; C^{0}\left(B_{R}, H\right)\right), 0, R_{2}\right), L_{m}\left(H^{\otimes m} ; H\right)\right)\right),
\end{aligned}
$$

(continuous and bounded on its domain) for each $n, l, 0<R_{2}<\infty$ and

$$
\lim _{n \rightarrow \infty} \sup _{0 \leq l \leq n}\left\|a_{n-l, l}\right\|_{C^{1}\left(V, C^{0}\left(B_{R_{1}} \times B\left(L^{q}\left(\Omega, \mathscr{F}, \lambda ; C^{0}\left(B_{R}, H\right)\right), 0, R_{2}\right), L_{n}\left(H^{\otimes n}, H\right)\right)\right)}=0
$$

for each $0<R_{1} \leq R$ when $0<R<\infty$, or each $0<R_{1}<R$ when $R=\infty$, for each $0<R_{2}<\infty$;

(ii) $\operatorname{ker}(E(t, \omega, \xi, y))=0$ for each $t, \xi$, and $y$, also for $\lambda$-almost every $\omega$;

(iii) $a_{y}(t, \omega, \xi, y)$ and $\partial a(t, \omega, \xi, y) / \partial y \in X_{0, d}(H):=\left\{z: S^{-1} z \in H_{d}\right\}$ and $\partial E(t, \omega, \xi, y) / \partial y \in L_{b}(H)$ for $\lambda$-almost all $\omega$ and each $t$, $\xi$, and $y$, where $H_{d}:=\left\{z: z \in H ; \sum_{j=1}^{\infty}\left|z_{j}\right|^{d}<\infty\right\}$ for each $0<d<\infty, H_{\infty}:=H$, with $d=b=\psi$ for $\mu_{S, \gamma, \psi} ; d=\infty$ and $b=0$ for the measure of the second type $\mu_{S}, z_{j}$ are the coordinates of the vector $z$ in the standard base in $H$; in addition for [20, Theorem 3.4(i)];

(iv) $\partial a_{l, k}(t, \omega, \xi, y) / \partial y \in L_{k+l, b}\left(H^{\otimes(k+l)} ; H\right)$ for each $l$ and each $k$ with either $b=\psi$ or $b=0$ correspondingly, where parameters $r, s$, and $q$ are the same as in [20, Theorems 3.3 and 3.4], respectively. The following theorem states the quasi-invariance of the transition measure $\mu^{F_{t, t_{0}}}\left(\left\{\omega: \xi\left(t_{0}, \omega, y\right)=0, \xi(t, \omega, y) \in A\right\}\right)=: P_{y}(A)$, where $F_{t, u}(\xi):=$ $\xi(t, \omega, y)-\xi(u, \omega, y)$.

THEOREM 2.5. Let either conditions (i), (ii), and (iii) or (i), (ii), (iii), and (iv) be satisfied, then the transition measure $P_{y}(A)$ of the stochastic process $\xi(t, \omega, y)$ being the solution of [20, (3.8) or Theorem 3.4(i)] and depending on the parameter $y \in V$ is quasi-invariant relative to each mapping $U\left(y_{2}, y ; \xi(t, \omega, y)\right):=$ $\xi\left(t, \omega, y_{2}\right)$ for each $y$ and $y_{2} \in V$.

Proof. The Kakutani theorem (see [4, Section II.4.1]) states that, whether $\prod_{k=1}^{\infty} \alpha_{k}$ converges to a positive number or diverges to zero, the measure $\mu$ is absolutely continuous or orthogonal with respect to $v$, correspondingly, where $\alpha_{k}:=\int_{X_{k}}\left(p_{k}\left(x_{k}\right)\right)^{1 / 2} v_{k}\left(d x_{k}\right), \mu_{k}$ is absolutely continuous relative to $v_{k}$, $\mu=\otimes_{k} \mu_{k}, v=\otimes_{k} \nu_{k}, \mu_{k}$ and $v_{k}$ are probability measures on measurable spaces $X_{k}$ for each $k \in \mathbb{N}, p_{k}(x):=\mu_{k}(d x) / \nu_{k}(d x)$. In the first case, $\prod_{k} p_{k}\left(x_{k}\right)$ converges in the mean to $\mu(d x) / v(d x)$. In the considered case here, let $X_{k}=\mathbf{K}$ for each $k \in \mathbb{N}$. Let $\mu_{k}(d x)=C f(x-y) v(d x)$ and $v_{k}(d x)=C f(x) v(d x)$, where $v$ is the nonnegative Haar measure on $\mathbf{K}, f$ is a positive function such that $f \in L^{1}(\mathbf{K}, v, \mathbb{R})$, and $C=$ const $>0$ such that $v(\mathbf{K})=1$. Then, $p_{k}(x)=$ $f(x-y) / f(x)$ and $\alpha_{k}=\int_{\mathbf{K}}(f(x-y) f(x))^{1 / 2} v(d x)$. For the $\psi$-Gaussian measure $f(x)=\int_{\mathrm{K}} \exp \left(-\beta|x|^{\psi}\right) \chi_{\gamma}(x) \chi_{1}(-z x) v(d x)$ (see [24] and [25, Section 7]). 
If $|y x| \leq 1$, then $\chi_{1}(y x)=1$. Therefore, there is a constant $C_{1}>0$ independent from $\beta$ and $\gamma$ such that $|f(z-y)-f(z)| \leq|f(z)|\left(1+C_{1} \exp \left(-\beta r^{-\psi}\right)\right)$ for each $y$ with $|y|<r$, where $\beta r^{-\psi}>1$, since due to Cauchy-Schwarz-Bunyakovskii inequality

$$
\begin{aligned}
& \left|\int_{|x|>1 / r} \exp \left(-\beta|x|^{\psi}\right) \chi_{\gamma}(x) \chi_{1}(-(z-y) x) v(d x)\right| \\
& \quad \leq\left|\int_{|x|>1 / r} \exp \left(-\beta|x|^{\psi}\right) \chi_{\gamma}(x) \chi_{1}(-z x) v(d x)\right| g(y, z) \\
& \quad \leq|f(z)| g(y, z)
\end{aligned}
$$

where

$$
g(y, z):=\left|\int_{|x|>1 / r} \exp \left(-\beta|x|^{\psi}\right) \chi_{\gamma}(x) \chi_{1}(-z x) \chi_{1}(2 y x) v(d x)\right| .
$$

Let $\left|y_{j} / v_{j}\right|=: r_{j}<1$ for each $j>j_{0}$, then $\left|\alpha_{j}-1\right| \leq C \exp \left(-\beta_{j} r_{j}^{-\psi}\right)$ for each $j>j_{0}$, where $C=$ const $>0$. In view of [21, Proposition 2.10] and the Kakutani theorem, $\mu_{S, \gamma, \psi}^{z}$ is equivalent to $\mu_{S, \gamma, \psi}$ for each $z \in X_{0, \psi}\left(C_{0}^{0}(T, H)\right)$, where $\mu^{z}(A):=\mu(A-z)$ for each Borel subset $A$ in $C_{0}^{0}(T, H)$, that is, $\mu_{S, \gamma, \psi}$ is quasiinvariant relative to shifts $z \in X_{0, \psi}\left(C_{0}^{0}(T, H)\right)$.

For the measure $\mu_{J}$ and $|y|<1 /|v|$, there is the equality $f((x-y) / v)=$ $f(x / v)$ for each $x \in \mathbf{K}$ and $0 \neq v \in \mathbf{K}$. In view of the definition of $f_{k}$, there is the equality

$$
\begin{aligned}
p_{k}(x)= & f_{k}\left(x-y_{k}\right) / f_{k}(x) \\
= & {\left[f\left(\left(x-y_{k}\right) / v_{k}\right) / f\left(x / v_{k}\right)\right] } \\
& \times\left[1+h_{k}\left(\left(x-y_{k}\right) / v_{k}\right) / f\left(\left(x-y_{k}\right) / v_{k}\right)\right] /\left[1+h_{k}\left(x / v_{k}\right) / f\left(x / v_{k}\right)\right] .
\end{aligned}
$$

If $\left|y_{k} / v_{k}\right| \leq 1$, then $f\left(\left(x-y_{k}\right) / v_{k}\right) / f\left(x / v_{k}\right)=1$ for each $x \in \mathbf{K}$. From the conditions imposed on $h_{k}$ and $f$, the Kakutani theorem, and [21, Proposition $2.10]$ it follows that $\mu_{S}$ is quasi-invariant relative to shifts $z \in X_{0, \infty}\left(C_{0}^{0}(T, H)\right)$.

The quasi-invariance factor $\rho(z, x):=\mu^{z}(d x) / \mu(d x)$ is Borel-measurable as follows from the construction of $\mu$, the Kakutani theorem, and the Lebesgue theorem about majorized convergence (see [6, Section 2.4.9]), since this is true for each of its one-dimensional projection. Banach theorem states that if $G$ is a topological group and $A \subset G$ is a Borel measurable set of second category, then $A \circ A^{-1}$ is a neighbourhood of the unit (see [3, Section 5.5]). The quasiinvariance factor satisfies the cocycle condition:

$$
\rho(z+h, x)=\rho(z, x-h) \rho(h, x)
$$

for each $z$ and $h \in X_{0, d}\left(C_{0}^{0}(T, H)\right)$ and each $x \in C_{0}^{0}(T, H)$. Therefore, in view of the Lusin theorem (see [6, Section 2.3.5]), $\rho(z, x):=\mu^{z}(d x) / \mu(d x)$ is such 
that $\mu\left(W_{L}\right)=1$ for each finite-dimensional subspace $L$ in $X_{0, d}\left(C_{0}^{0}(T, H)\right)$, where either $\mu=\mu_{S, \gamma, \psi}$ or $\mu=\mu_{S}, W_{L}:=\{x: \rho(z, x)$ is defined and continuous by $z \in L\}$.

In view of the preceding consideration, $\lim _{n \rightarrow \infty} \rho\left(\hat{P}_{n} z, x\right)=\rho(z, x)$ for $\mu$ almost all $x \in C_{0}^{0}(T, H)$, moreover, this convergence is uniform by $z$ in each ball $B(L, 0, c)$ for each finite-dimensional subspace $L$ in $X_{0, s}\left(C_{0}^{0}(T, H)\right)$, where $\hat{P}_{n}$ is a projection on a subspace $\operatorname{sp}_{\mathbf{K}}\left(e_{1}, \ldots, e_{n}\right)=\mathbf{K}^{n}$, where $\left\{e_{j}: j\right\}$ is the orthonormal base in $X_{0, s}\left(C_{0}^{0}(T, H)\right)$. Evidently, $X_{0, s}\left(C_{0}^{0}(T, H)\right)$ is dense in $C_{0}^{0}(T, H)$.

Stochastic antiderivational [20, (3.8)] is the particular case of [20, Theorem 3.4(i)]. Therefore, it is sufficient to consider the latter equation. Below, it is shown that the one-parameter family of solutions $\xi(t, \omega, y)$ is of class $C^{1}$ by $y \in V$. Let $X_{0}(t, y)=x(y), \ldots$,

$$
\begin{aligned}
X_{n}(t, y)= & x(y) \\
+ & \sum_{m+b=1}^{\infty} \sum_{l=0}^{m}\left(\hat { P } _ { u ^ { b + m - l } , w ( u , \omega ) l } \left[a_{m-l+b, l}\left(u, X_{n-1}(u, \omega, y), y\right)\right.\right. \\
& \left.\left.\circ\left(I^{\otimes b} \otimes a^{\otimes(m-l)} \otimes E^{\otimes l}\right)\right]\right)\left.\right|_{u=t} .
\end{aligned}
$$

Consequently,

$$
\begin{gathered}
X_{n+1}(t, y)-X_{n}(t, y) \\
=\sum_{m+b=1}^{\infty} \sum_{l=0}^{m}\left(\hat { P } _ { u ^ { b + m - l } , w ( u , \omega ) ^ { l } } \left[a_{m-l+b, l}\left(u, X_{n}(u, y), y\right)\right.\right. \\
\left.-a_{m-l+b, l}\left(u, X_{n-1}(u, y), y\right)\right] \\
\left.\circ\left(I^{\otimes b} \otimes a^{\otimes(m-l)} \otimes E^{\otimes l}\right)\right)\left.\right|_{u=t},
\end{gathered}
$$

where $t_{j}=\sigma_{j}(t)$ for each $j=0,1,2, \ldots$, for the shortening of the notation, $X_{n}$, $x$, and $a_{l, k}$ are written without the argument $\omega ; a$ and $E$ are written without their variables. Then

$$
\begin{aligned}
& M \sup _{y} \| \hat{P}_{u^{b+m-l}, w(u, w) l}\left[a_{m-l+b, l}\left(u, X_{n}(u, y), y\right)\right. \\
&\left.-a_{m-l+b, l}\left(u, X_{n-1}(u, y), y\right)\right]\left.\right|_{\left(B_{R_{1}} \times B\left(L^{q}, 0, R_{2}\right) \times V\right)} \\
&\left.\quad \circ\left(I^{\otimes b} \otimes a^{\otimes(m-l)} \otimes E^{\otimes l}\right)\right|_{u=t} \|^{g} \\
& \leq K\left(M\left\|\hat{P}_{u^{b+m-l}, w(u, w) l}\right\|^{g}\right)\left\|\left.a_{m-l+b, l}\right|_{\left(B_{R_{1}} \times B\left(L^{q}, 0, R_{2}\right) \times V\right)}\right\|^{g} \\
& \times\left(M \sup _{u, y}\left\|X_{n}(u, y)-X_{n-1}(u, y)\right\|^{g}\right)\left(M \sup _{u, y}\|a\|^{m-l}\right)\left(M \sup _{u, y}\|E\|^{l}\right),
\end{aligned}
$$


where $X_{n} \in C_{0}^{0}\left(B_{R}, H\right)$ for each $\omega, y \in V$ and for each $n, K$ is the same constant as in [20, Theorem 3.4], $1 \leq \mathfrak{g}<\infty$. On the other hand,

$$
\begin{aligned}
X_{1}(t, y)= & x(t, y) \\
+ & \sum_{m+b=1}^{\infty} \sum_{l=0}^{m}\left(\hat { P } _ { u ^ { b + m - l } , w ( u , \omega ) l } \left[a_{m-l+b, l}(u, x(u, y), y)\right.\right. \\
& \left.\left.\circ\left(I^{\otimes b} \otimes a^{\otimes(m-l)} \otimes E^{\otimes l}\right)\right]\right)\left.\right|_{u=t},
\end{aligned}
$$

consequently,

$$
\begin{aligned}
& \left\|X_{1}(t, y)-X_{0}(t, y)\right\|^{g} \\
& \leq \sup _{m, l, b}\left(\| \hat{P}_{u^{b+m-l}, w(u, \omega)^{l}}\left[a_{m-l+b, l}(u, x(u, y), y)\right.\right. \\
& \left.\left.\circ\left(I^{\otimes b} \otimes a^{\otimes(m-l)} \otimes E^{\otimes l}\right)\right]\right)\left.\right|_{u=t} \|^{g} .
\end{aligned}
$$

Due to condition (ii) for each $\epsilon>0$ and $0<R_{2}<\infty$ there exists $B_{\epsilon} \subset B_{R}$ such that

$$
\begin{aligned}
& K \sup _{m, l, b}\left(\|\left.\hat{P}_{u^{b+m-l}, w(u, w)} l\right|_{B_{\epsilon}}[\right.\left.a_{m-l+b, l}(u, *, y)\right|_{\left(B_{\epsilon} \times B\left(L^{q}, 0, R_{2}\right) \times V\right)} \\
&\left.\left.\circ\left(I^{\otimes b} \otimes a^{\otimes(m-l)} \otimes E^{\otimes l}\right)\right] \|\right)^{g}=: c<1 .
\end{aligned}
$$

On the other hand, the partial difference quotient has the continuous extension $\bar{\Phi}^{1}\left(X_{n+1}-X_{n}\right)(y ; h ; \zeta)$, that is expressible through $\bar{\Phi}^{1}$ of $a_{l, k}, a$, and $E$, and also through $a_{l, k}, a$, and $E$ themselves, where $y \in V, h \in Y, \zeta \in \mathbf{K}$ such that $y+\zeta h \in V$, since analogous to $\left(X_{n+1}-X_{n}\right)$ estimates are true for $\bar{\Phi}^{1}\left(X_{n+1}-\right.$ $\left.X_{n}\right)$. Therefore, there exists the unique solution on each $B_{\epsilon}$ and it is of class $C^{1}$ by $y \in V$, since $\sup _{u, y} \max \left(\left\|X_{1}(u, y)-X_{0}(u, y)\right\|_{L^{q}(\Omega, H)}, \| \bar{\Phi}^{1}\left(X_{1}(u, y)-\right.\right.$ $\left.\left.X_{0}(u, y)\right) \|_{L^{q}(\Omega, H)}\right)<\infty$, and $\lim _{l \rightarrow \infty} c^{l} C=0$ for each $C>0$, hence there exists $\lim _{n \rightarrow \infty} X_{n}(t, y)=X(t, y)=\left.\xi(t, \omega, y)\right|_{B_{\epsilon}}$, where

$$
\begin{aligned}
C:=M \sup _{u \in B_{\epsilon}, y \in V} \max & \left(\left\|X_{1}(u, y)-X_{0}(u, y)\right\|_{L^{q}(\Omega, H)}^{q},\right. \\
& \left.\left\|\bar{\Phi}^{1}\left(X_{1}(u, y)-X_{0}(u, y)\right)\right\|_{L^{q}(\Omega, H)}^{q}\right) \leq(c+1) K<\infty,
\end{aligned}
$$

here $B_{\epsilon}$ is an arbitrary ball of radius $\epsilon$ in $B_{R}, t \in B_{\epsilon}$. Therefore, $\xi(t, \omega, y) \in$ $C^{1}\left(V, L^{q}\left(\Omega, \mathscr{F}, \lambda ; C^{0}\left(B_{R}, H\right)\right)\right)$.

From [20, Proposition 3.11] it follows that the multiplicative operator functional $T(t, v ; \omega ; y)$ is of class $C^{1}$ by the parameter $y \in V$ such that $\xi(t, \omega, y)=$ $T(t, v ; \omega ; y) \xi(v, \omega, y)$ for each $t$ and $v \in T$.

Due to the existence and uniqueness of the solution $\xi(t, \omega, y)$ for each $y \in V$, there exists the operator $U\left(y_{2}, y ; \xi(t, \omega, y)\right):=\xi\left(t, \omega, y_{2}\right)$, that may be nonlinear by $\xi$. The variation of the family of solutions $\{\xi(t, \omega, y): y\}$ corresponds to the differential $D_{y} \xi(t, \omega, y)$. Since $\xi(t, \omega, y)$ is of class $C^{1}$ by $y$, 
then $U\left(y_{2}, y ; \xi(t, \omega, y)\right)$ is of class $C^{1}$ by $y$ and $y_{2}$. The operator $U\left(y_{2}, y ; *\right)$ has the inverse, since $U\left(y, y_{2} ; U\left(y_{2}, y ; \xi(t, \omega, y)\right)\right)=\xi(t, \omega, y)$ for each $y_{2}$ and $y \in V, t \in T$, and $\omega \in \Omega$. Therefore, $U^{-1}\left(y_{2}, y\right.$; * $)$ is also of class $C^{1}$ by $y_{2}$ and $y$. In view of conditions (iii), (iv) and $\xi\left(t, \omega, y_{2}\right)-\xi(t, \omega, y) \in X_{0, d}(H)$. On the other hand, either $\mu_{S, \gamma, \psi}$ or $\mu_{S}$ is quasi-invariant relative to shifts $z \in$ $X_{0, d}\left(C_{0}^{0}(T, H)\right)$ and $S=S_{1} \otimes S_{2}$, consequently, the transition measure $P_{y}$ is quasi-invariant relative to shifts $z \in X_{0, d}(H)$. In view of conditions (ii), (iii), and (iv), $\partial U\left(y_{2}, y ; \eta\right) / \partial \eta-I \in L_{b}(H)$ for each $y_{2}$ and $y \in V$, where $\eta \in\{\xi(t, \omega, y)$ : $y$, either $b=\psi$ or $b=0$, respectively. Since $\mu_{S}\left(C_{0}^{0}(T, H)\right)=1$, then $P_{y}(H)=1$, hence $U\left(y_{2}, y ; *\right)$ is defined $P_{y}$-almost everywhere on $H$ for each $y_{2}$ and $y \in V$. Therefore, there exists $n$ such that for each $j>n$ the mappings $V(j ; x):=$ $x+P_{j}\left(U^{-1}(x)-x\right)$ and $U(j ; x):=x+P_{j}(U(x)-x)$ are invertible and $\lim _{j}\left|\operatorname{det} U^{\prime} x(j ; x)\right|=\left|\operatorname{det} U_{x}^{\prime}(x)\right|$ and $\lim _{j}\left|\operatorname{det} V_{x}^{\prime}(j ; x)\right|=1 /\left|\operatorname{det} U_{x}^{\prime}(x)\right|$, where $U(x):=U\left(y_{2}, y ; x\right), y_{2}$, and $y \in V$.

In view of [10, Theorem 3.28] for each $y_{2}$ and $y \in V$, the transition measures $P_{y_{2}}$ and $P_{y}$ are equivalent.

THEOREM 2.6. Let conditions (i), (ii), (iii), and (iv) in Section 2.1 be satisfied and let $\phi$ be a $C^{1}$-diffeomorphism of a subset $V$ clopen in $\mathrm{K}$ onto the unit ball $B(\mathbf{K}, 0,1)$. Then

(1) the transition measure $P_{y}$ corresponding to $\mu_{S, \gamma, d}$ is pseudodifferentiable by the parameter $y=\phi(z)$ of order $b \in \mathbb{C}$ for each $\operatorname{Re}(b) \geq 0$, where $z \in V$;

(2) $P_{y}$ corresponding to $\mu_{S}$ with $h_{k}$ such that $\sum_{k} \delta_{k}<\infty$, where $\delta_{k}:=$ $\sup _{x \in B(\mathrm{~K}, 0,1)}\left|\mathrm{PD}_{c}\left(b, h_{k}(x)\right)\right|$, is pseudodifferentiable by the parameter $y=\phi(z)$ of order $b$ for each $b \geq 0$, moreover, $P_{y}$ is pseudodifferentiable for each $b \in C$, when each $f_{k}$ is locally constant, that is, $h_{k}=0$ for each $k \in \mathbb{N}$.

Proof. Up to a constant multiplier, the operator $\mathrm{PD}_{c}(b, h(x))$ of $[18$, Section I.3.1] coincides with the pseudodifferential operator

$$
D^{b}\left(h(x) \mathrm{Ch}_{B(\mathbf{K}, 0,1)}(x)\right)
$$

from [25, Section 9], where $\mathrm{Ch}_{A}$ is the characteristic function of the subset $A$ in $\mathbf{K}$. If $\psi \in L^{2}(\mathbf{K}, w, \mathbb{C})$ and $b>0$, then due to the Cauchy-Schwarz-Bunyakovskii inequality there exists

$$
\int_{\mathbf{K} \backslash B(\mathbf{K}, x, 1)}[\psi(x)-\psi(y)]|x-y|^{-1-b} w(d y),
$$

where $w$ is the Haar nonnegative measure on $\mathbf{K}$. Then,

$$
F\left[D^{b}(h(x))\right]=|x|^{b} F[h(x)],
$$

where $F(h)(x):=\int_{\mathbf{K}} h(y) \chi_{1}((x, y)) w(d x)$ is the Fourier transform (see also [21, Section 3.4]). In view of [25, Theorem 7.4], the Fourier transform $f \mapsto F[f]$ 
is the bijective continuous isomorphism of $L^{2}(\mathbf{K}, w, \mathbb{C})$ onto itself such that $f(x)=\lim _{r \rightarrow \infty} \int_{B(\mathbf{K}, 0, r)} F[f](y) \chi_{1}(-(y, x)) w(d y)$ and $(f, g)=(F[f], F[g])$ for each $f, g \in L^{2}(\mathbf{K}, w, \mathbb{C})$. If $F[\psi](x)=C \exp \left(-\beta|x|^{d}\right) \chi_{\gamma}(x)$, then there exists $D^{b} \psi(x)$ for each $b \geq 0$. In accordance with [25, Example 4.3.9], $\int_{\mathbf{K}} \chi_{\gamma}(x) w(d x)$ $=0$ for each $\gamma \neq 0$. In view of [25, Example 4.3.10],

$$
\int_{\mathrm{Q}_{p}}|x|^{n d} \chi_{1}(y x) w(d x)=\left[1-p^{n d}\right]\left[1-p^{-n(d+1)}\right]^{-1}|y|^{-n(d+1)}
$$

for each $d \in \mathbb{C}$ with $\operatorname{Re}(d)>0$ and $n \in\{1,2,3, \ldots\}$.

If $f$ is a locally constant function as in Note 2.4 , then $\operatorname{PD}_{c}(b, f)$ exists for each $b \in \mathbb{C}$. On the other hand, $\mathrm{PD}_{c}\left(b, f+h_{k}\right)=\mathrm{PD}_{c}(b, f)+\mathrm{PD}_{c}\left(b, h_{k}\right)$.

Let $g$ be a continuously differentiable function $g: \mathbb{R} \rightarrow \mathbb{R}$ such that

$$
\|g\|_{C^{1}(\mathbb{R}, \mathbb{R})}:=\sup _{x}|g(x)|+\sup _{x}\left|g^{\prime}(x)\right|<\infty,
$$

that is, $g \in C_{b}^{1}(\mathbb{R}, \mathbb{R})$. If for $f: \mathbf{K} \rightarrow \mathbb{R}$ and $x \in \mathbf{K}$, there exists $[f(x)-f(y)] \mid x-$ $\left.y\right|^{-1-b} \in L^{1}(\mathbf{K}, w, \mathbb{C})$ as the function by $y \in \mathbf{K}$, then

$$
\begin{aligned}
& \int_{\mathbf{K}}[g \circ f(x)-g \circ f(y)]|x-y|^{-1-b} w(d y) \\
& =\int_{S(f, x)}[g \circ f(x)-g \circ f(y)][f(x)-f(y)]^{-1}[f(x)-f(y)]|x-y|^{-1-b} w(d y),
\end{aligned}
$$

where $S(f, x):=\{y: y \in \mathbf{K}, f(x) \neq f(y)\}$, consequently, there exists $\operatorname{PD}(b, g \circ$ $f)(x)$.

If instead of $g$ there exists $h \in C^{1}(\mathbf{K}, \mathbf{K})$ such that

$$
\|h\|_{C^{1}(\mathbf{K}, \mathbf{K})}:=\max \left(\sup _{x}|h(x)|, \sup _{x, y}\left|\bar{\Phi}^{1} h(x ; 1 ; y)\right|\right)<\infty
$$

that is $h \in C_{b}^{1}(\mathbf{K}, \mathbf{K})$, then

$$
\begin{aligned}
\int_{\mathbf{K}}[f \circ h(x)-f \circ h(y)]|x-y|^{-1-b} w(d y) \\
=\int_{S(h, x)}[f \circ h(x)-f \circ h(y)]|h(x)-h(y)|^{-1-b} \\
\quad \times|h(x)-h(y)|^{1+b}|x-y|^{-1-b} w(d y)
\end{aligned}
$$

exists, hence there exists $\operatorname{PD}(b, f \circ h)(x)$. Analogous two statements are true for the operator $\mathrm{PD}_{c}$ instead of PD.

In view of [25, equation 9.1.5], $D^{\alpha} D^{\beta} \psi=D^{\beta} D^{\alpha} \psi=D^{\alpha+\beta} \psi$ for each $\alpha \neq-1$, $\beta \neq-1$, and $\alpha+\beta \neq-1$ for each $\psi \in D^{\prime}$ such that there exist $D^{\alpha} \psi, D^{\beta} \psi$, and $D^{\alpha+\beta} \psi$, where $D^{\prime}$ is the topologically dual space to the space $D$ of locally constant functions $\phi: \mathbf{K} \rightarrow \mathbb{R}$. On the other hand, $D$ is dense in $D^{\prime}$ in the 
weak topology (see [25, Section 6]). Evidently, $L^{2} \cap D$ is dense in $L^{2}(\mathbf{K}, w, \mathbb{R})$ also. The characteristic functional of the Gaussian measure belongs to $D^{\prime}$ and is locally constant on $\mathbf{K} \backslash\{0\}$. Due to [25, Sections 7.2 and 7.3], the Fourier transform is the linear topological isomorphism of $D$ on $D$ and of $D^{\prime}$ on $D^{\prime}$. Then, $\mu_{S, y, d}^{g}(d x) / w(d x) \in L^{1}(\mathbf{K}, w, \mathbb{R}) \cap D^{\prime}$ for each $g \in C_{0}^{0}(T, H)^{*}$.

In view of [11, Theorem 4.3] and using the Kakutani theorem as in Note 2.4, we get the statements of this theorem, since the quasi-invariance factor $P_{y}(d x) / P_{u}(d x)$ is pseudodifferentiable as the function by $y$ of order $b$ for each fixed $u \in B(\mathbf{K}, 0,1)$.

THEOREM 2.7. Let $G$ be either a loop group or a diffeomorphism group defined as in [18, Section II.2.1] and Remark 2.3 above, then there exists a stochastic process $\xi(t, \omega)$ on $G$ which induces a quasi-invariant transition measure $P$ on $G$ relative to $G^{\prime}$, and $P$ is pseudodifferentiable of order $b$ for each $b \in \mathbb{C}$ such that $\operatorname{Re}(b) \geq 0$ relative to $G^{\prime}$, where a dense subgroup $G^{\prime}$ is given in $[18$, Section II.2.4] and Remark 2.3 above.

Proof. These topological groups also have structures of $C^{\infty}$-manifolds, which are infinite-dimensional over the local field $\mathbf{K}$, but they do not satisfy the Campbell-Hausdorff formula in any open local subgroup [16, 18]. Their manifold structures and actions of $G^{\prime}$ on $G$ will be sufficient for the construction of the desired measures. These separable Polish groups have embeddings as clopen subsets into the corresponding tangent Banach spaces $Y^{\prime}$ and $Y$ in accordance with $[14,18]$ and Remark 2.3, where $Y^{\prime}$ is the dense subspace of $Y$. As usually $T G=\bigcup_{x \in G} T_{x} G$ and $T_{x} G=(x, Y)$.

Let $G$ be a complete separable relative to its metric $\rho C^{\infty}$-manifold on a Banach space $Y$ over $\mathbf{K}$ such that it has an embedding into $Y$ as the clopen subset. Let $\tau_{G}: T G \rightarrow G$ be a tangent bundle on $G$. It is trivial, since $T G=G \times Y$ for the considered case here. Let $\theta: Z_{G} \rightarrow G$ be a trivial bundle on $G$ with the fibre $Z$ such that $Z_{G}=Z \times G$, then $L_{1,2}\left(\theta, \tau_{G}\right)$ will be an operator bundle with a fibre $L_{1,2}(Z, Y)$ (see [21, Section 2.4]). Let $\Pi:=\tau_{G} \oplus L_{1,2}\left(\theta, \tau_{G}\right)$ be a Whitney sum of bundles $\tau$ and $L_{1,2}\left(\theta, \tau_{G}\right)$.

Since $G$ is clopen in $Y$, the valuation group of $\mathbf{K}$ is discrete in $(0, \infty)$, then it has a clopen disjoint covering by balls $B\left(Y, x_{j}, r_{j}\right)$, that is, the atlas $\operatorname{At}(G)$ of $G$ has a refinement $\operatorname{At}^{\prime}(G)$ being a disjoint atlas.

On $Y$, consider the measure $\mu_{S, \gamma, d}$ or $\mu_{S}$ as in Note 2.4. Then in view of [21, Theorem 4.2] and [20, Section 2.1], there exists the stochastic process $w(t, \omega)$ corresponding to $\mu_{S, \gamma, d}$ or $\mu_{S}$ (see also [21, Definition 4.1] and [20, Section 3.1]). Suppose that $f$ and $h_{k}$, for each $k \in \mathbb{N}$ defining the measure $\mu_{S}$, satisfy the conditions of Note 2.4 and of Theorem 2.6.

Now, let $G$ be a loop or a diffeomorphism group of the corresponding manifolds over the field $\mathbf{K}$. Consider for $G$ a field $U$ with a principal part $\left(a_{\eta}, E_{\eta}\right)$, where $a_{\eta} \in T_{\eta} G$ and $E_{\eta} \in L_{1,2}\left(H, T_{\eta} G\right)$ and $\operatorname{ker}\left(E_{\eta}\right)=\{0\}, \theta: H_{G} \rightarrow G$ is a trivial bundle with a Banach fiber $H$ and $H_{G}:=G \times H, L_{1,2}\left(\theta, \tau_{\eta}\right)$ is an operator bundle 
with a fibre $L_{1,2}\left(H, T_{\eta} G\right)$ such that $\left(a_{\eta}, E_{\eta}\right)$ satisfies conditions of [20, Theorem 3.3]. For [20, Theorem 3.4(i)], we take additionally $\left(a_{l, k}\right)_{\eta}$ for each $l, k$ satisfy the conditions of [20, Theorem 3.4]. To satisfy conditions of quasi-invariance and pseudodifferentiability of transition measures theorems, we choose $a_{\eta}$, $E_{\eta}$, and $\left(a_{k, l}\right)_{\eta}$ of class $C^{1}$ and satisfy conditions (iii) and (iv) in Section 2.1 by $y:=\eta \in G^{\prime}=: V$ for each $k$ and $l$.

We can take initially $\mu_{I, \gamma, d}$ or $\mu_{I}$ as a cylindrical measure on a Banach space $X^{\prime}$ such that $T_{\eta} G^{\prime} \subset X^{\prime} \subset T_{\eta} G$. If $A_{\eta}$ is the $L_{d}$-operator or the $L_{1}$-operator with $\operatorname{ker}\left(A_{\eta}\right)=\{0\}$, then $A_{\eta}$ gives the $\sigma$-additive measure $\mu_{A_{\eta}, A_{\eta}^{*} z, d}$ or $\mu_{A_{\eta}}$ in the completion $X_{1, \eta}^{\prime}$ of $X^{\prime}$ with respect to the norm $\|x\|_{1}:=\left\|A_{\eta} x\right\|$ (see Note 2.4).

There exists the solution $\xi(t, \omega, \eta)=\xi_{\eta}(t, \omega)$ of stochastic antiderivational equation [20, (3.8) or Theorem 3.4(i)]. When the embedding $\theta$ of $T_{\eta} G^{\prime}$ into $T_{\eta} G$ is $\theta=\theta_{1} \theta_{2}$ with $\theta_{1}$ and $\theta_{2}$ of class $L_{d}$ for $\mu_{S, \gamma, d}$ or of class $L_{1}$ for $\mu_{S}$, then there exists $A_{\eta}$ such that $\mu_{A_{\eta}, A_{\eta} z, d}$ or $\mu_{A_{\eta}}$ is the quasi-invariant and pseudodifferentiable of order $b$ measure on $T_{\eta} G$ relative to shifts on vectors from $T_{\eta} G^{\prime}$ (see Theorems 2.5 and 2.6). Henceforth, we impose such demand on $A_{\eta}$ for each $\eta \in G^{\prime}$.

Consider left shifts $L_{h}: G \rightarrow G$ such that $L_{h} \eta:=h \circ \eta$. We take $a_{e} \in T_{e} G^{\prime}, A_{e} \in$ $L_{1, d}\left(T_{e} G^{\prime}, T_{e} G\right)$, or $A_{e} \in L_{1,1}\left(T_{e} G^{\prime}, T_{e} G\right)$, respectively, $\left(a_{k, l}\right)_{\eta} \in L_{k+l}\left(\left(T_{e} G\right)^{\otimes(k+l)}\right.$; $T_{e} G$ ) for each $k$ and each $l$, where $H, T_{e} G^{\prime}$, and $T_{e} G$ in their own norm uniformities are isomorphic with $c_{0}\left(\omega_{0}, \mathbf{K}\right)$. Then, we put $a_{x}=\left(D L_{x}\right) a_{e}$ and $A_{x}=\left(D L_{x}\right) \circ A_{e}$ for each $x \in G$, hence $a_{x} \in T_{e} G$ and $A_{x} \in L_{1, k}\left(H_{x},\left(D L_{x}\right) T_{e} G\right)$, where $\left(D L_{x}\right) T_{e} G=T_{x} G$ and $T_{e} G^{\prime} \subset T_{e} G, H_{x}:=\left(D L_{x}\right) T_{e} G^{\prime}, \kappa=d$ or $\kappa=1$. Operators $L_{h}$ are (strongly) $C^{\infty}$-differentiable diffeomorphisms of $G$ such that $D_{h} L_{h}: T_{\eta} G \rightarrow T_{h \eta} G$ is correctly defined, since $D_{h} L_{h}=h_{*}$ is the differential of $h$. In view of the choice of $G^{\prime}$ in $G$, each partial difference quotient $\bar{\Phi}^{n} L_{h}\left(X_{1}, \ldots, X_{n} ; \zeta_{1}, \ldots, \zeta_{n}\right)$ is of class $C^{0}$ and $D^{n} L_{h}$ is of class $L_{n+1, \kappa}\left(T G^{\prime \otimes n} \times\right.$ $\left.G^{\prime}, T G\right)$ for each vector fields $X_{1}, \ldots, X_{n}$ on $G^{\prime}, \zeta_{1}, \ldots, \zeta_{n} \in \mathbf{K}$ with $\zeta_{j} p_{2}\left(X_{j}\right)+$ $h \in G^{\prime}$ and $h \in G^{\prime}$, since for each $0 \leq l \in \mathbb{Z}$ the embedding of $T^{l} G^{\prime}$ into $T^{l} G$ is the product of two operators of the $L_{d}$-class or the embedding is of the $L_{1}$-class, where $T^{0} G:=G, X=\left(x, X_{x}\right) \in T_{x} G, x \in G^{\prime}, X_{x} \in Y^{\prime}, p_{1}(X)=x, p_{2}(X)=X_{x}$. Take a dense subgroup $G^{\prime}$ from Note 2.2 or Remark 2.3 correspondingly and consider left shifts $L_{h}$ for $h \in G^{\prime}$.

The considered groups $G$ are separable, hence the minimal $\sigma$-algebra generated by cylindrical subalgebras $f^{-1}\left(\mathscr{B}_{n}\right), n=1,2, \ldots$, coincides with the $\sigma$ algebra $\mathscr{B}$ of Borel subsets of $G$, where $f: G \rightarrow \mathbf{K}^{n}$ are continuous functions, $\mathscr{B}_{n}$ is the Borel $\sigma$-algebra of $\mathbf{K}^{n}$. Moreover, $G$ is the topological Radon space (see [4, Theorem I.1.2 and Proposition I.1.7]). Let

$$
P\left(t_{0}, \psi, t, W\right):=P\left(\left\{\omega: \xi\left(t_{0}, \omega\right)=\psi, \xi(t, \omega) \in W\right\}\right)
$$

be the transition probability of the stochastic process $\xi$ for $t \in T$, which is defined on a $\sigma$-algebra $\mathscr{B}$ of Borel subsets in $G, W \in \mathscr{B}$, since each measure 
$\mu_{A_{\eta}, A_{\eta}^{*} z, q}$ is defined on the $\sigma$-algebra of Borel subsets of $T_{\eta} G$ (see above). On the other hand, $T(t, \tau, \omega) g x=g T(t, \tau, \omega) x$ is the stochastic evolution family of operators for each $\tau \neq t \in T$. There exists the transition measure $P\left(t_{0}, \psi, t, W\right)$ such that it is a $\sigma$-additive quasi-invariant and pseudodifferentiable of order $b$ relative to the action of $G^{\prime}$ by the left shifts $L_{h}$ on $\mu$ measure on $G$, for example, $t_{0}=0$ and $\psi=e$ with the fixed $t_{0} \in T$ (see [18, Remark I.3.3 and Definition I.3.4]).

NoTE 2.8. In Theorem 2.7, $G^{\prime}$ is on the Banach space $Y^{\prime}$ and $G$ on the Banach space $Y$ over $\mathbf{K}$ such that $G^{\prime}$ and $G$ are complete relative to their uniformities $\cup_{G^{\prime}}$ and $\mathcal{U}_{G}$. There are inclusions $T G^{\prime}=G^{\prime} \times Y^{\prime} \subset G \times Y^{\prime} \subset G \times Y=T G$. The completion of $T G^{\prime}$ relative to the uniformity $\mathcal{U}_{G} \times U_{Y^{\prime}}$ produces the uniform space $G \times Y^{\prime}$. Therefore, each $\varkappa_{G} \times \vartheta_{Y^{\prime}}$-uniformly continuous vector field $X=$ $\left(x, X_{x}\right)$ on $G^{\prime}$ has the unique extension on $G$ such that $X_{x} \in Y^{\prime}$ for each $x \in G$

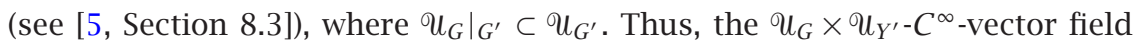
$X$ on $G^{\prime}$ has the $\bigcup_{G} \times \cup_{Y^{\prime}}-C^{\infty}$ extension on $G$ and it provides the 1-parameter

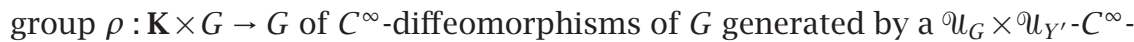
vector field $X_{\rho}$ on $G^{\prime}$ (see $\left.[12,13]\right)$, that is, $\left.(\partial \rho(v, x) / \partial v)\right|_{v=0}=X_{\rho}(x)$ for each $x \in G$, where $v \in \mathbf{K}, X_{\rho}(x) \in G \times Y^{\prime}$. In view of Theorem 2.7, the transition measure $P$ is quasi-invariant and pseudodifferentiable of order $b$ relative to the 1-parameter group $\rho$.

This approach is also applicable to the case of two Polish manifolds $G^{\prime}$ and $G$ of class $C^{\infty}$ on $Y^{\prime}$ and $Y$ over $\mathbf{K}$. The quasi-invariance and pseudodifferentiability of the measure $P$ on $G$ relative to the 1-parameter group $\rho$ (by the

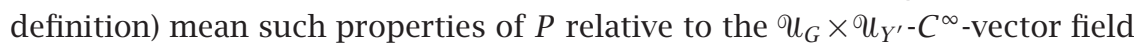
$X$ on $G^{\prime}$.

Evidently, considering different $(a, E)$ and $\left\{a_{k, l}: k, l\right\}$, we see that there exist $\mathscr{C}=\operatorname{card}(\mathbb{R})$ nonequivalent stochastic (in particular, Wiener) processes on $G$ and $\mathscr{C}$ orthogonal quasi-invariant pseudodifferentiable of order $b \in \mathbb{C}$ with $\operatorname{Re}(b)>0$ measures on $G$ relative to $G^{\prime}$.

If $M$ is compact, then in the case of the diffeomorphism group, its dense subgroup $G^{\prime}$ can be chosen such that $G^{\prime} \supset \operatorname{Diff}\left(t^{\prime}, M\right)$ for $\operatorname{dim}_{\mathbf{K}} M=n \in \mathbb{N}$ and $t^{\prime}=t+s$ for $0 \leq t \in \mathbb{R}, s>n v, v=\operatorname{dim}_{\mathrm{Q}_{p}}(\mathbf{K})$. Analogously, the manifold $M \subset B\left(\mathbf{K}^{n}, 0, r\right)$ and the group $G:=\operatorname{Diff}\left(a n_{r}, M\right)$ of analytic diffeomorphisms $f: M \rightarrow M$ can be considered having analytic extensions on $B(\mathbf{K}, 0, r)$ with the corresponding norm topology, where $r>0$ and $r<\infty$. Then, there exists the stochastic process $\xi$ on $T_{e} G$ such that it generates the transition measure $P$ on $T_{e} G$, its restriction on the clopen subset $G$ embedded into $T_{e} G$ produces the quasi-invariant and pseudodifferentiable, each of order $b \in \mathbb{C}$ with $\operatorname{Re}(b) \geq 0$ measure $\left.P\right|_{G}$ relative to the dense subgroup $G^{\prime}:=\operatorname{Diff}\left(a n_{R}, M\right)$ for $R>r>0$, since the embedding $T_{e} G^{\prime}$ into $T_{e} G$ is of class $L_{1}$ (see also Theorem 2.9).

THEOREM 2.9. Let $G$ be a separable Banach-Lie group over a local field $\mathbf{K}$. Then there exists a probability quasi-invariant and pseudodifferentiable, each 
of order $b \in \mathbb{C}$ with $\operatorname{Re}(b)>0$, transition measure $P$ on $G$ relative to a dense subgroup $G^{\prime}$ such that $P$ is associated with a non-Archimedean stochastic process.

Proof. We consider the following two cases: (I) $G$ locally satisfies the Campbell-Hausdorff formula; (II) $G$ does not satisfy it in any neighbourhood of $e$ in $G$. The first case permits to describe $G^{\prime}$ more concretely. There exists the embedding of $G$ into $T_{e} G$ as the clopen subset, since $G$ is the Polish group. The second case can be considered quite analogously to Theorem 2.7, where the dense subgroup $G^{\prime}$ can be characterized by the condition that the embedding of $T_{e} G^{\prime}$ into $T_{e} G$ is $\theta=\theta_{1} \theta_{2}$ with $\theta_{1}$ and $\theta_{2}$ of class $L_{d}$ or $\theta$ of class $L_{1}$, where $d=\psi$ or $d=1$ for stochastic processes associated either with $\mu_{S, \gamma, \psi}$ or $\mu_{S}$, respectively.

It remains to consider the first case. For $G$, there exist a Banach-Lie algebra $g$ and the exponential mapping exp : $V \rightarrow U$, where $V$ is a neighbourhood of 0 in $g$ and $U$ is a neighbourhood of $e$ in $G$ such that $\exp (V)=U$, where $\exp (X+Y)=\exp (X) \exp (Y)$ for commuting elements $X$ and $Y$ of $g$, that is, $[X, Y]=0, \exp (X) Y \exp (-X)=\exp (\operatorname{ad} X) Y, \exp (\lambda X)=\sum_{j=0}^{\infty} \lambda^{j} X^{j} / j !$, $V=B(g, 0, r)$ is a ball of radius $0<r<\infty$ in $g, \lambda \in \mathbf{K}, \lambda X \in V, g=T_{e} G$. The radii of convergence of the exponential and Hausdorff series corresponding to $\log (\exp (X) \cdot \exp (Y))$ are positive such that for each $0<R<p^{1 /(1-p)}$ to a ball $B(g, 0, R)$ there corresponds a clopen subgroup $G_{1}$ supplied with the Hausdorff function (see [2, Sections II.6 and II.8]). Therefore, the exponential mapping supplies $G$ with the structure of the analytic manifold over $\mathbf{K}$. The analytic atlas of $\mathbb{N}$ is denoted by $\operatorname{At}(G)=\left\{\left(U_{j}, \phi_{j}\right): j \in \mathbb{N}\right\}$, that is, $\phi_{j}: U_{j} \rightarrow V_{j}$ are diffeomorphisms of $U_{j}$ onto $V_{j}$, where $U_{j}$ and $V_{j}$ are clopen in $G$ and in $g$, respectively, connecting mappings $\phi_{j} \circ \phi_{i}^{-1}$ are analytic on $\phi_{i}\left(U_{i} \cap U_{j}\right) \subset g$. Therefore, the exponential mapping provides $G$ with the covariant derivation $\nabla$ and a bilinear tensor $\Gamma$ such that $\nabla_{X} Y={ }_{L} \nabla_{X} Y-{ }_{L} T(X, Y) / 2$, where the leftinvariant derivation on $G$ is defined by ${ }_{L} \nabla_{X} \tilde{Y}=0$ for an arbitrary left-invariant vector field $\tilde{Y}$ and all vector fields $X$ on $G$, a vector field $\tilde{Y}$ is called left-invariant if $T L_{g} \tilde{Y}(h)=\tilde{Y}(g h), L_{g} h:=g h$ for each $g, h \in G, T L_{g}$ is the tangent mapping of $L_{g}, \nabla_{X} Y_{u}=D Y_{u} \cdot X_{u}+\Gamma_{u}\left(X_{u}, Y_{u}\right)$. For such $\nabla$, the torsion tensor is zero (see [9, Section 1.7], [18], and [25, Section 14.7]). It defines the rigid analytic geometry and the corresponding atlas on $G$. Nevertheless, $\operatorname{At}(G)$ has the refinement $\operatorname{At}^{\prime}(G)$ such that charts of $\operatorname{At}^{\prime}(G)$ compose the disjoint covering of $G$.

Let $a_{x}$ be an analytic vector field and $A_{x}$ be an analytic operator field on $G$ such that $A_{x}$ is an injective compact operator of class $L_{d}$ for each $x \in G$, since $\boldsymbol{g}$ is of separable type over a spherically complete field $\mathbf{K}$ and hence isomorphic with $c_{0}\left(\omega_{0}, \mathbf{K}\right)$ (see [24, Chapter 5]), where $d=\psi$ or $d=1$. Let $w_{x}(t, \omega)$ be a non-Archimedean stochastic (or, in particular, Wiener) process in $T_{x} G$ such that $a_{x} t+A_{x} w_{x}(t) \in T_{x} G$, since the space $C_{0}^{0}$ is isomorphic with $c_{0}$. For a ball $B_{R}:=B(\mathbf{K}, 0, R)$ in $\mathbf{K}$ for $0<R<\infty$, let $B\left(\mathbf{K}, t_{j}, r\right)$ be a disjoint paving for 
sufficiently small $0<r<\infty$ for which

$$
\xi_{x}^{q}(t)=\exp _{\xi_{x, k}^{q}}\left\{a_{\xi_{x, k}^{q}}\left(t-t_{k}\right)+A_{\xi_{x, k}^{q}}\left[w_{\xi_{x, k}^{q}}(t)-w_{\xi_{x, k}^{q}}\left(t_{k}\right)\right]\right\}
$$

is defined, where $\xi_{x, k}^{q}=\xi_{x}^{q}\left(t_{k}\right)$ for $k=0,1, \ldots, n, \xi_{x}^{q}(0)=x, q$ denotes the partition of $B_{R}$ into $B\left(\mathbf{K}, t_{j}, r\right)$. Then there exists the process $\xi=\lim _{q} \xi^{q}(t)$ which is by our definition a solution of the following stochastic equation:

$$
d \xi(t, \omega)=\exp _{\xi(t, \omega)}\left\{a_{\xi(t, \omega)} d t+A_{\xi(t, \omega)} d w(t, \omega)\right\}
$$

for $t \in B_{R}$. A function $f(t, x)$ such that $f(t, \xi):=\ln _{\xi(t, \omega)} \xi(t, \omega)$ satisfies the condition of [21, Theorem 4.7] on the corresponding domain $W$, where $(t, x) \in$ $W \subset T \times H$. In view of [21, Theorem 4.7] after coordinate mapping of a chart $(U, \phi)$, this equation takes the following form on $g$ :

$$
\begin{gathered}
\phi(\xi(t, \omega))=\phi\left(\xi\left(t_{0}, \omega\right)\right)+\left.\left(\hat{P}_{u} a_{\xi(u)}^{\phi}\right)\right|_{u=t}+\left.\left(\hat{P}_{w_{\xi(u)}^{\phi}(u, \omega)} E\right)\right|_{u=t} \\
-\sum_{m=2}^{\infty}(m !)^{-1} \sum_{l=0}^{m}\left(\begin{array}{c}
m \\
l
\end{array}\right)\left(\hat { P } _ { u ^ { m - l , w _ { \xi ( u ) } ^ { \phi } ( u , \omega ) ^ { l } } } \left[\left(\frac{\partial^{m-2} \Gamma_{\phi(\xi(u))}^{\phi}}{\partial x^{m-2}}\right)\right.\right. \\
\left.\left.\circ\left(a_{\xi(u)}^{\phi}(u)^{\otimes(m-l)} \otimes E^{\otimes l}\right)\right]\right)\left.\right|_{u=t},
\end{gathered}
$$

where $E=A_{\xi(u, \omega)}^{\phi}, a^{\phi}=\left(\partial \phi_{x} / \partial x\right) a_{x}, A_{x}^{\phi}=\left(\partial \phi_{x} / \partial x\right) A_{x}\left(\partial \phi_{x}^{-1} / \partial x\right)$, since

$$
\begin{array}{ll}
h^{\phi}=\left(\frac{\partial g^{\phi}}{\partial x}\right) f^{\phi}+\Gamma_{\phi(x)}^{\phi}\left(f^{\phi}, g^{\phi}\right) & \text { for } h=\nabla_{f} \mathcal{g}, \\
f^{\phi}=\left(\frac{\partial \phi}{\partial x}\right) f, \quad g^{\phi}=\left(\frac{\partial \phi}{\partial x}\right) g, & h^{\phi}=\left(\frac{\partial \phi}{\partial x}\right) h,
\end{array}
$$

$\Gamma^{\phi}$ is a bilinear operator of Christoffel in $g$, which has the transformation property $D\left(\psi \circ \phi^{-1}\right) \cdot \Gamma_{\phi(x)}^{\phi}=D^{2}\left(\psi \circ \phi^{-1}\right)+\Gamma_{\psi(x)}^{\psi} \circ\left(D\left(\psi \circ \phi^{-1}\right) \times D\left(\psi \circ \phi^{-1}\right)\right)$ such that $\nabla_{X} Y_{\phi}=D Y_{\phi} \cdot X_{\phi}+\Gamma_{\phi(x)}^{\phi}\left(X_{\phi}, Y_{\phi}\right), \Gamma_{\phi(x)}^{\phi}$ denotes $\Gamma$ for the chart $(U, \phi), \psi$ corresponds to another chart $(V, \psi)$ such that $U \cap V \neq \varnothing, f, g$, and $h$ are vector fields, since $\left[\partial\left(\psi \circ \phi^{-1}\right) / \partial t\right]=0$, that is, [21, Corollary 4.6] is applicable instead of [21, Theorem 4.7] because $f$ corresponds to $\left(\psi \circ \phi^{-1}\right)$ (see [1], [9, Section 1.5]). Since $a_{x}$ and $A_{x}$ are analytic, then $a$ and $E$ satisfy the conditions of [20, Theorem 3.3].

The function $\Gamma$ is analytic on the corresponding domain. On the other hand, $g$ is isomorphic with $c_{0}\left(\omega_{0}, \mathbf{K}\right)$ as the Banach space. If $Z$ is the center of $g$, then ad $: g / Z \rightarrow g l\left(c_{0}\left(\omega_{0}, \mathbf{K}\right)\right)$ is the injective representation, where $g l\left(c_{0}\right)$ denotes the general linear algebra on $c_{0}, \operatorname{ad}(x) y:=[x, y]$ for each $x, y \in g$. Since $Z$ is commutative, it also has an injective representation in $g l\left(c_{0}\right)$, consequently, $g$ has an embedding into $g l\left(c_{0}\left(\omega_{0}, \mathbf{K}\right)\right)$, since $c_{0} \oplus c_{0}$ is isomorphic 
with $c_{0}$. Therefore, each $x \in g$ can be written in the form $x=\sum_{i, j} x^{i, j} X_{i, j}$, where $\left\{X_{i, j}: i, j \in \mathbb{N}\right\}$ is the orthonormal basis of $g$ as the Banach space, $x^{i, j} \in \mathbf{K}$, $\lim _{i+j \rightarrow \infty} x^{i, j}=0$, consequently, $g$ has an embedding into $L_{0}\left(c_{0}\left(\omega_{0}, \mathbf{K}\right)\right)$. Then, $\Gamma$ can be written in local coordinates $x_{s(i, j)}:=x^{i, j}$, where $s: \mathbb{N}^{2} \rightarrow \mathbb{N}$ is a bijection for which $\lim _{i+j \rightarrow \infty} s(i, j)=\infty, X_{i, j}=: q_{s(i, j)}$, since

$$
\left(x^{n}\right)^{j, k}=\sum_{l_{1}, \ldots, l_{n-1}} x^{j, l_{1}} x^{l_{1}, l_{2}} \cdots x^{l_{n-1}, k} X_{j, k}
$$

when $X_{j, k}=\left(\delta_{a, j} \delta_{b, k}: a, b \in \mathbb{N}\right), \psi \circ \phi^{-1}(x)=\sum_{s} q_{s} a_{m}^{s} x^{m}$ with $a_{m}^{s} \in \mathbf{K}$ and $\lim _{s+|m|+\operatorname{Ord}(m) \rightarrow \infty} a_{m}^{s}=0$, since $\exp (x)$ has a radius of convergence $0<\tilde{r}=$ $p^{-1}$ for the characteristic $\operatorname{char}(\mathbf{K})=0$ (see [23, Theorem 25.6]), where $m=$ $\left(m_{1}, \ldots, m_{k}\right), k=\operatorname{Ord}(m), 0 \leq m_{1} \in \mathbb{Z}, \ldots, 0 \leq m_{k-1} \in \mathbb{Z}, 0<m_{k} \in \mathbb{Z}, 0 \leq k \in \mathbb{Z}$. Evidently, there exists $0<r<\infty$ such that the series for $\psi \circ \phi^{-1}$ converges in $B\left(c_{0}, 0, r\right)$ for $V \cap U \neq \varnothing$. Hence, each $a_{m-l, l}:=\left(\partial^{m-2} \Gamma_{\phi(x)}^{\phi} / \partial^{m-2} x\right) / m$ ! for $m \geq 2$ and $a_{1,0}=a_{0,1}=(\partial \phi / \partial x)$ satisfies [20, Theorem 3.4(ii)]. Due to [20, Theorem 3.4] there exists the unique solution of [20, Theorem 3.4(i)]. Consider $G^{\prime}$ corresponding to $g^{\prime}$ such that the embedding $\theta$ of $g^{\prime}$ into $g$ is of class $L_{1}$ for $\mu_{S}$ or $\theta=\theta_{1} \theta_{2}$, where $\theta_{1}$ and $\theta_{2}$ are of class $L_{\psi}$ for $\mu_{S, \gamma, \psi}$.

Let $T \in L_{d}(g)$ or $T=T_{1} T_{2}$ with $T_{1}$ and $T_{2} \in L_{d}(g)$, where $d=1$ or $d=\psi$. Consider $h_{1}:=T(g), h_{2}:=\operatorname{sp}_{\mathbf{K}}\left(\left[h_{1}, g\right] \cup h_{1}\right)$ and by induction $h_{n+1}:=\operatorname{sp}_{\mathbf{K}}\left(\left[h_{n}, g\right] \cup\right.$ $h_{n}$ ), then $h_{n+1} \supset h_{n}$ and $h_{n}$ is the subalgebra in $g$ for each $n \in \mathbb{N}$. In view of [21, Proposition 2.10], the space $L_{d}(g)$ is the ideal in $L(g)$. Therefore, $h:=\bigcup_{n} h_{n}$ is the ideal in $g$ due to the anticommutativity and the Jacobi identity. Since $\mathbf{K}$ is spherically complete, there exists $h_{n+1} \ominus h_{n}=: t_{n+1}$ for each $n \in \mathbb{N}$ and $t_{1}:=h_{1}$ such that $t_{n}$ is the $\mathbf{K}$-linear subspace of $g$ (see [24]). The completion in $g$ of vectors $z$ is denoted by $\mathscr{C}_{0}\left(g,\left\{t_{n}: n\right\}\right)=: y$ such that $z=\sum_{n} z_{n}$ with $z_{n} \in t_{n}$ for each $n$ and $\lim _{n \rightarrow \infty} z_{n}=0$. Evidently, $y$ is the proper ideal in $g$ such that $h \subset y$, since $g$ is infinite dimensional over $\mathbf{K}$. Then, the embedding $\theta$ of $y$ into $g$ is of class either $L_{1}$ or $\theta=\theta_{1} \theta_{2}$ such that $\theta_{1}$ and $\theta_{2}$ belong to $L_{d}$.

Due to this, let $a$ and $A$ be such that $\left[a_{x}, y\right] \subset y$ and $\left[A_{x}, \operatorname{ad}(y)\right] \subset \operatorname{ad}(y)$ for each $x \in G$, where $\operatorname{ad}(x) g:=[x, g]$ for each $x, g \in g$, that is, $\operatorname{ad}(x) \in L(g)$. If $g \in y \cap V$, then $\exp (\operatorname{ad}(g))-I$ is either of the class $L_{1}$ or of the product of two operators each of which is of the class $L_{d}$.

There exists a countable family $\left(g_{j}, W_{j}\right): j \in \mathbb{N}$ of elements $g_{j} \in G \backslash W$ for each $j>1$ and clopen subsets $e \in W_{j} \subset W$ such that $g_{1}=e, W_{1}=W$, and $\left\{g_{j} W_{j}: j\right\}$ is a locally finite covering of $G$, since $G$ is separable and ultrametrizable (see [5, Section 5.3]). If $P$ is a quasi-invariant and pseudodifferentiable of order $b$ measure on a clopen subgroup $W$ relative to a dense subgroup $W^{\prime}$, then $P(S):=\left(\sum_{j} P\left(\left(g_{j}^{-1} S\right) \cap W_{j}\right) 2^{-j}\right)\left(\sum_{j} P\left(W_{j}\right) 2^{-j}\right)^{-1}$ for each Borel subset $S$ in $G$ is quasi-invariant and pseudodifferentiable of order $b$ measure on $G$ relative to the dense subgroup $G^{\prime}:=\bigcup_{j} g_{j}\left(W_{j} \cap W^{\prime}\right)$. The group $G$ 
is totally disconnected and is left-invariantly ultrametrizable (see [8, Section 8 and Theorem 5.5] and [5, Section 6.2]), consequently, in each neighbourhood of $e$ there exists a clopen subgroup in $G$. Then, conditions of Section 2.1 and Theorem 2.6 are satisfied. Therefore, analogously to Theorem 2.7, there are $S, \gamma$, and the stochastic process corresponding to $\mu_{S, \gamma, \psi}$ or $\mu_{S}$ such that the transition measure $P$ is quasi-invariant and pseudodifferentiable relative to $G^{\prime}$.

2.2. Theorem 2.7 gives the subgroup $G^{\prime}$ concretely for the given group $G$, but Theorem 2.9 describes concretely $G^{\prime}$ only for the case of $G$ satisfying the Campbell-Hausdorff formula. For a Banach-Lie group not locally satisfying the Campbell-Hausdorff formula, Theorem 2.9 gives only the existence of $G^{\prime}$.

These transition measures $P=: v$ on $G$ induce strongly continuous unitary regular representations of $G^{\prime}$ given by the following formula: $T_{h}^{v} f(g):=$ $\left(v^{h}(d g) / v(d g)\right)^{1 / 2} f\left(h^{-1} g\right)$ for $f \in L^{2}(G, v, \mathbb{C})=: H, T_{h}^{v} \in U(H), U(H)$ denotes the unitary group of the Hilbert space $H$. For the strong continuity of $T_{h}^{v}$ the continuity of the mapping $G^{\prime} \ni h \mapsto \rho_{v}(h, g) \in L^{1}(G, v, \mathbb{C})$ and that $v$ is the Borel measure are sufficient, where $g \in G$, since $G$ is the Polish space and hence the Radon space (see [4, Theorem I.1.2]). On the other hand, the continuity of $\rho_{v}(h, g)$ by $h$ from the Polish group $G^{\prime}$ into $L^{1}(G, v, \mathbb{C})$ follows from $\rho_{v}(h, g) \in L^{1}(G, v, \mathbb{C})$ for each $h \in G^{\prime}$ and $G^{\prime}$ being the topological subgroup of $G$ (see [3, 7]).

Then, analogously to Theorem 2.7 there can be constructed quasi-invariant and pseudodifferentiable measures on the manifold $M$ relative to the action of the diffeomorphism group $G_{M}$ such that $G^{\prime} \subset G_{M}$. Then, Poisson measures on configuration spaces associated with either $G$ or $M$ can be constructed [19]. There exists the stochastic process corresponding to $\mu_{S, \gamma, \psi}$ with the certain choice of $a, E$, and $a_{k, l}$ such that the regular representation is irreducible, for the stochastic process corresponding to $\mu_{S}$ the family of $\left\{f_{k}: k\right\}$ and $a, E$, and $a_{k, l}$ can be taken such that the regular representation is irreducible.

More generally, it is possible to consider instead of the group $G$ a Polish topological space $X$ on which $G^{\prime}$ acts jointly continuously, $\phi:\left(G^{\prime} \times X\right) \ni(h, x) \mapsto$ $h x=: \phi(h, x) \in X, \phi(e, x)=x$ for each $x \in X, \phi(v, \phi(h, x))=\phi(v h, x)$ for each $v$ and $h \in G^{\prime}$ and each $x \in X$. If $\phi$ is the Borel function, then it is jointly continuous [3, 7].

THEOREM 2.10. Let $X$ be an infinite Polish topological space with a $\sigma$-additive $\sigma$-finite nonnegative nonzero ergodic Borel measure $v$ with $\operatorname{supp}(v)=X$ and quasi-invariant relative to an infinite dense in itself Polish topological group $G^{\prime}$ acting on $X$ by the Borel function $\phi$. If

(i) $\operatorname{sp}_{\mathbb{C}}\left\{\psi \mid \psi(g):=\left(v^{h}(d g) / v(d g)\right)^{1 / 2}, h \in G^{\prime}\right\}$ is dense in $H$,

(ii) for each $f_{1, j}$ and $f_{2, j}$ in $H, j=1, \ldots, n, n \in \mathbb{N}$, and each $\epsilon>0$ there exists $h \in G^{\prime}$ such that $\left|\left(T_{h} f_{1, j}, f_{2, j}\right)\right| \leq \epsilon\left|\left(f_{1, j}, f_{2, j}\right)\right|$, when $\left|\left(f_{1, j}, f_{2, j}\right)\right|>0$, then the regular representation $T: G^{\prime} \rightarrow U(H)$ is topologically irreducible. 
Proof. From condition (i), it follows that the vector $f_{0}$ is cyclic, where $f_{0} \in$ $H$ and $f_{0}(g)=1$ for each $g \in X$. In view of $\operatorname{card}(X) \geq \mathrm{N}_{0}$ and the ergodicity of $v$ for each $n \in \mathbb{N}$, there are subsets $U_{j} \in B f(X)$ and $g_{j} \in G^{\prime}$ such that $v\left(\left(g_{j} U_{j}\right) \cap\right.$ $\left.\left(\bigcup_{i=1, \ldots, j-1, j+1, \ldots, n} U_{i}\right)\right)=0$ and $\prod_{j=1}^{n} v_{j}\left(U_{j}\right)>0$. Together with condition (ii), this implies that there is no finite-dimensional $G^{\prime}$-invariant subspace $H^{\prime}$ in $H$ such that $T_{h} H^{\prime} \subset H^{\prime}$ for each $h \in G^{\prime}$ and $H^{\prime} \neq\{0\}$. Hence, if there is a $G^{\prime}$-invariant closed subspace $H^{\prime} \neq 0$ in $H$, it is isomorphic with the subspace $L^{2}(V, v, \mathbb{C})$, where $V \in B f(X)$ with $v(V)>0$.

Each Polish space is Čech-complete. By the Baire-category theorem in a Čechcomplete space $X$, the union $A=\bigcup_{i=1}^{\infty} A_{i}$ of a sequence of nowhere dense subsets $A_{i}$ is a codense subset (see [5, Theorem 3.9.3]). On the other hand, in view of [8, Theorem 5.8], a subgroup of a topological group is discrete if and only if it contains an isolated point. Therefore, we can choose a Radon probability measure $\lambda$ on $G^{\prime}$ such that $\lambda$ has no atoms and $\operatorname{supp}(\lambda)=G^{\prime}$. In view of the strong continuity of the regular representation, there exists the Bochner integral $\int_{X} T_{h} f(g) v(d g)$ for each $f \in H$, which implies its existence in the weak Pettis sense. The final part of the proof, follows from [18, Section II.3.2] (see also $[16,19])$.

THEOREM 2.11. On a loop or a diffeomorphism group $G$, there exists a stochastic process, which generates a quasi-invariant measure $\mu$ relative to a dense subgroup $G^{\prime}$ such that the associated regular unitary representation $T^{\mu}: G^{\prime} \rightarrow$ $U\left(L^{2}(G, \mu, \mathbb{C})\right)$ is irreducible.

Proof. Pseudodifferentiable measures of order $l$ can be used, either for each $l \in \mathbb{N}$ or for each $-l \in \mathbb{N}$, for the verification of Theorem 2.10(i). Transition measures corresponding to stochastic processes that are quasi-invariant and pseudodifferentiable, each of order $b \in \mathbb{C}$ with $\operatorname{Re}(b) \leq 0$, can be analogously constructed starting with the corresponding measures $\mu_{S}$. To satisfy the conditions of this theorem, for example, in Theorem 2.7, it can be taken $a=0, E$ nondegenerate independent from $t$, and each $a_{k, l}=0$ besides $a_{0,1}=1$; in Theorem 2.9 it can be taken $a=0, E$ nondegenerate independent from $t$, and $a_{k, l}$ is defined by the exponential mapping for $G$.

From the construction of $G^{\prime}$ and $\mu$ in Theorem 2.7, it follows that if a function $f \in L^{1}(G, \mu, \mathbb{C})$ satisfies the following condition: $f^{h}(g)=f(g)(\bmod \mu)$ by $g \in G$ for each $h \in G^{\prime}$, then $f(x)=\operatorname{const}(\bmod \mu)$, where $f^{h}(g):=f(h g)$, $g \in G$.

In view of Theorem 2.7 and $[18,21]$ and references therein, the stochastic process on the Banach manifold $G$ induces the stochastic process on the Banach space $T_{e} G$ with the help of the manifold non-Archimedean exponential locally affine mapping. Then, the left action $L_{h}$ of $G^{\prime}$ on $G$ induces the local left action of $G^{\prime}$ on a neighbourhood $V$ of 0 in $T_{e} G$ with $v(V)>0$, where $v$ is induced by $\mu$. A class of compact subsets approximates from below each measure $\mu^{f}, \mu^{f}(d g):=|f(g)| \mu(d g)$, where $f \in L^{2}(G, \mu, \mathbb{C})=: H$. Finally, we get from 
Theorem 2.7 that there exists $\mu$, which is ergodic, and that conditions (i) and (ii) of Theorem 2.10 are satisfied. Evidently, $G^{\prime}$ and $G$ are infinite and dense in themselves. Hence, from Theorem 2.10 the statement of this theorem follows.

Notes 2.12. In view of [22, Proposition II.1] for the separable Hilbert space $H$, the unitary group endowed with the strong operator topology $U(H)_{s}$ is the Polish group. Let $U(H)_{n}$ be the unitary group with the metric induced by the operator norm. In view of the Pickrell's theorem (see [22, Section II.2]), if $\pi: U(H)_{n} \rightarrow U(V)_{s}$ is a continuous representation of $U(H)_{n}$ on the separable Hilbert space $V$, then $\pi$ is also continuous as a homomorphism from $U(H)_{s}$ into $U(V)_{s}$. Therefore, if $T: G^{\prime} \rightarrow U(H)_{s}$ is a continuous representation, then there are new representations $\pi \circ T: G^{\prime} \rightarrow U(V)_{s}$. On the other hand, the unitary representation theory of $U(H)_{n}$ is the same as that of $U_{\infty}(H):=$ $U(H) \cap\left(1+L_{0}(H)\right)$, since the group $U_{\infty}(H)$ is dense in $U(H)_{s}$.

Two theorems about induced representations of the dense subgroups $G^{\prime}$ were proved in [12], which are also applicable to the cases considered here.

\section{Stochastic antiderivational equations and measures on a loop monoid and a path space}

THEOREM 3.1. On the monoid $G=\Omega \xi(M, N)$ from [18, Part I] and for each $b_{0} \in \mathbb{C}$ with $\operatorname{Re}\left(b_{0}\right) \geq 0$, there exists a stochastic process $\eta(t, \omega)$ on $G$ such that the transition measure $P$ is quasi-invariant and pseudodifferentiable each of order $b \in \mathbb{C}$ with $\operatorname{Re}(b) \geq \operatorname{Re}\left(b_{0}\right)$ relative to the dense submonoid $G^{\prime}:=\Omega_{\xi}^{\{k\}}(M, N)$ from Note 2.2 (with $c>0$ and $c^{\prime}>0$ ).

Proof. In view of [18, Lemma I.2.17], it is sufficient to consider the case of $M$ with the finite atlas $\mathrm{At}^{\prime}(M)$. The rest of the proof is quite analogous to that of Theorem 2.7 using the definitions of the quasi-invariance and the pseudodifferentiability for semigroups from [18].

DEFINITION AND Note 3.2. In view of [18, Definition and Note I.2.9], each space $N^{\xi}$ has the additive group structure when $N=B(Y, 0, R), 0<R \leq \infty$.

Therefore, the factorization by the equivalence relation $K_{\xi} \times$ id produces the monoid of paths $C_{0}^{\theta}(\xi, \bar{M} \rightarrow N) /\left(K_{\xi} \times\right.$ id $)=: S_{\xi}(M, N)$ in which compositions are defined not for all elements, where $y_{1}$ id $y_{2}$ if and only if $y_{1}=y_{2} \in N$. There exists a composition $f_{1} f_{2}=\left(g_{1} g_{2}, y\right)$ if and only if $y_{1}=y_{2}=y$, where $f_{i}=\left(g_{i}, y_{i}\right), g_{i} \in \Omega_{\xi}(M, N)$, and $y_{i} \in N^{\xi}, i \in\{1,2\}$. The latter semigroup has elements $e_{y}$ such that $f=e_{y} \circ f=f \circ e_{y}$ for each $f$ when their composition is defined, where $y \in N^{\xi}, f=(g, y), g \in \Omega_{\xi}(M, N), e_{y}=(e, y)$. If $N^{\xi}$ is a monoid, then $S_{\xi}(M, N)$ can be supplied with the structure of a direct product of two monoids. Therefore, $P_{\xi}(M, N):=L_{\xi}(M, N) \times N^{\xi}$ is called the path group.

THEOREM 3.3. On the path group $G=P_{\xi}(M, N)$ from Definition and Note 3.2 when $N=B(Y, 0, R)$ and $N^{\xi}$ is supplied with the additive group structure, and 
each $b_{0} \in \mathbb{C}$ with $\operatorname{Re}\left(b_{0}\right) \geq 0$, there exists a stochastic process $\eta(t, \omega)$ for which a transition measure $P$ is quasi-invariant and pseudodifferentiable each of order $b \in \mathbb{C}$ with $\operatorname{Re}(b) \geq \operatorname{Re}\left(b_{0}\right)$ relative to a dense subgroup $G^{\prime}$.

Proof. Since $P_{\xi}(M, N)=L_{\xi}(M, N) \times N^{\xi}$, it is sufficient to construct two stochastic processes on $L_{\xi}(M, N)$ and $N^{\xi}$ and to consider transition measures for them. In view of Theorems 2.7 and 2.9, the desired processes and transition measures for them exist.

DEFINITION 3.4. Let the topology of $\Omega_{\xi}(M, N)$ be defined relative to countable $\operatorname{At}(M)$. If $F$ is the free Abelian group corresponding to $\Omega_{\xi}(M, N)$, then there exists a set $\bar{W}$ generated by formal finite linear combinations over $\mathbb{Z}$ of elements from $C_{0}^{0}\left(\xi,\left(M, s_{0}\right) \rightarrow\left(N, y_{0}\right)\right)$ and a continuous extension $\bar{K}_{\xi}$ of $K_{\xi}$ onto $W_{\xi}(M, N)$ and a subset $\bar{B}$ of $\bar{W}$ generated by elements $[f+g]-[f]-[g]$ such that $W_{\xi}(M, N) / \bar{K}_{\xi}$ is isomorphic with $L_{\xi}(M, N)$, where

$$
W_{\xi}(M, N):=\bar{W} / \bar{B},
$$

$f$ and $g \in C_{0}^{0}\left(\xi,\left(M, s_{0}\right) \rightarrow\left(N, y_{0}\right)\right),[f]$ is an element in $\bar{W}$ corresponding to $f, \bar{W}$ is in a topology inherited from the space $C_{0}^{0}\left(\xi,\left(M, s_{0}\right) \rightarrow\left(N, y_{0}\right)\right)^{\mathbb{Z}}$ in the Tychonoff product topology. We call $W_{\xi}(M, N)$ an $O$-group. Clearly, the composition in $C_{0}^{0}\left(\xi,\left(M, s_{0}\right) \rightarrow\left(N, y_{0}\right)\right)$ induces the composition in $W_{\xi}(M, N)$. Then, $W_{\xi}(M, N)$ is not the algebraic group, but associative compositions are defined for its elements due to the homomorphism $\chi^{*}$ given by [18, formulas I.2.6.2.(5,6)], hence $W_{\xi}(M, N)$ is the monoid without the unit element.

Let $\mu_{h}(A):=\mu(h \circ A)$ for each $A \in B f\left(W_{\xi}(M, N)\right)$ and $h \in W_{\xi}(M, N)$, then as in $[18$, Sections I.3.3, 3.4] we get the definition of quasi-invariant and pseudodifferentiable measures.

Now let $G^{\prime}:=W_{\xi}^{\{k\}}(M, N)$ be generated by $C_{0,\{k\}}^{0}\left(\xi,\left(M, s_{0}\right) \rightarrow(N, 0)\right)$ as in [18, Section II.5.1], then it is the dense $O$-subgroup in $W_{\xi}(M, N)$, where $c>0$ and $c^{\prime}>0$.

THEOREM 3.5. Let $G:=W_{\xi}(M, N)$ be the O-group as in Definition 3.4, At $(M)$ finite, and $b_{0} \in \mathbb{C}$ with $\operatorname{Re}\left(b_{0}\right) \geq 0$. Then there exists a stochastic process $\eta(t, \omega)$ on $G$ for which the transition measure $P$ is quasi-invariant and pseudodifferentiable each of order $b \in \mathbb{C}$ with $\operatorname{Re}(b) \geq \operatorname{Re}\left(b_{0}\right)$ on $G$ relative to a dense $O$-subgroup $G^{\prime}$.

Proof. The uniform space $C_{0}^{0}(\xi, M \rightarrow N)$ has the embedding as the clopen subset into $C_{0}^{0}\left(\xi, M \rightarrow Y\right.$ ) (see [14]). Here, we can take $a \in T G^{\prime}$ and $A \in L_{1, s}(\theta, \tau)$ without relations with $D L_{h}$, where $s=q$ or $s=1$, respectively. Then, repeating the major parts of the proof of Theorem 2.7 without $L_{h}$ and so more simply, but using actions of vectors fields of $T G^{\prime}$ by $\rho_{X}$ on $G$, we get the statement of this theorem, since $\left(D_{X} \rho_{X}\right) Y$ and $\left[\left(\nabla_{X}\right)^{n}\left(D_{X} \rho_{X}\right)\right] Y$ are products of two operators of class $L_{n+2, q}\left(\left(T G^{\prime}\right)^{n+2}, T G\right)$ and also of class $L_{n+2,1}\left(\left(T G^{\prime}\right)^{n+2}, T G\right)$ for each $C^{\infty}$-vector fields $X$ and $Y$ on $G^{\prime}$ and for each $n \in \mathbb{N}$. In view of Note 2.8, 
there exists a stochastic process $\eta(t, \omega)$ for which the transition measure $P$ is quasi-invariant and pseudodifferentiable relative to each 1-parameter diffeomorphism group of $G^{\prime}$ associated with a $U_{G} \times U_{Y^{\prime}}-C^{\infty}$-vector field on $G^{\prime}$.

\section{REFERENCES}

[1] N. Bourbaki, Éléments de mathématique. Fasc. XXXIII. Variétés différentielles et analytiques. Fascicule de résultats (Paragraphes 1 à 7), Actualités Scientifiques et Industrielles, no. 1333, Hermann, Paris, 1967 (French).

[2] Lie Groups and Algebras, Izdat. Mir, Moscow, 1976 (Russian).

[3] J. P. R. Christensen, Topology and Borel Structure, North-Holland Publishing, Amsterdam, 1974.

[4] Yu. L. Dalecky and S. V. Fomin, Measures and Differential Equations in InfiniteDimensional Space, Mathematics and Its Applications (Soviet Series), vol. 76, Kluwer Academic Publishers, Dordrecht, 1991.

[5] R. Engelking, General Topology, Mir, Moscow, 1986 (Russian).

[6] H. Federer, Geometric Measure Theory, Die Grundlehren der mathematischen Wissenschaften, vol. 153, Springer-Verlag, New York, 1969.

[7] F. Fidaleo, Continuity of Borel actions of Polish groups on standard measure algebras, Atti Sem. Mat. Fis. Univ. Modena 48 (2000), no. 1, 79-89.

[8] E. Hewitt and K. A. Ross, Abstract Harmonic Analysis. Vol. I: Structure of Topological Groups. Integration Theory, Group Representations, Die Grundlehren der Mathematischen Wissenschaften, vol. 115, Academic Press, New York, 1963.

[9] W. Klingenberg, Riemannian Geometry, de Gruyter Studies in Mathematics, vol. 1, Walter de Gruyter, Berlin, 1982.

[10] S. V. Ludkovsky, The non-Archimedean analogs of the Bochner-Kolmogorov, Minlos-Sazonov and Kakutani theorems, preprint, 2000, http://arxiv.org/ abs/math.FA/0010230.

[11] _-, Quasi-invariant and pseudo-differentiable measures on a nonArchimedean Banach space. I. Real-valued measures, preprint, 2001, http://arxiv.org/abs/math.GM/0106169 (see also short version: Analysis Mathem. (2002) 287-316).

[12] _ Stochastic processes on geometric loop groups and diffeomorphism groups of real and complex manifolds, associated unitary representations, preprint, 2001, http://arxiv.org/abs/math.GR/0102222.

[13] _ Measures on groups of diffeomorphisms of non-Archimedean Banach manifolds, Russian Math. Surveys 51 (1996), no. 2, 338-340.

[14] — Embeddings of non-Archimedean Banach manifolds into nonArchimedean Banach spaces, Russ. Math. Surv. 53 (1998), no. 5(323), 1097-1098.

[15] _ Quasi-invariant measures on non-Archimedean semigroups of loops, Russian Math. Surveys 53 (1998), no. 3, 633-634.

[16] _ Measures on groups of diffeomorphisms of non-Archimedean manifolds, representations of groups and their applications, Theoret. and Math. Phys. 119 (1999), no. 3, 698-711.

[17] _ Properties of quasi-invariant measures on topological groups and associated algebras, Ann. Math. Blaise Pascal 6 (1999), no. 1, 33-45.

[18] _ Quasi-invariant measures on non-Archimedean groups and semigroups of loops and paths, their representations. I, II, Ann. Math. Blaise Pascal 7 (2000), no. 2, 19-53, 55-80. 
[19] _ _ Poisson measures for topological groups and their representations, Southeast Asian Bull. of Math. 25 (2002), 653-680.

[20] Stochastic antiderivational equations on non-Archimedean Banach spaces, Int. J. Math. Math. Sci. 2003 (2003), no. 41, 2587-2602.

[21] _ Stochastic processes on non-Archimedean Banach spaces, Int. J. Math. Math. Sci. 2003 (2003), no. 21, 1341-1363.

[22] K.-H. Neeb, On a theorem of S. Banach, J. Lie Theory 7 (1997), no. 2, 293-300.

[23] W. H. Schikhof, Ultrametric Calculus, Cambridge Studies in Advanced mathematics, vol. 4, Cambridge University Press, Cambridge, 1984.

[24] A. C. M. van Rooij, Non-Archimedean Functional Analysis, Monographs and Textbooks in Pure and Applied Math., vol. 51, Marcel Dekker, New York, 1978.

[25] V. S. Vladimirov, I. V. Volovich, and E. I. Zelenov, p-Adic Analysis and Mathematical Physics, Nauka, Moscow, 1994 (Russian).

S. V. Ludkovsky: Theoretical Department, Institute of General Physics, Russian Academy of Sciences, 38 Vavilov Street, Moscow 119991, GSP-1, Russia

E-mail address: 1udkovsk@fp1.gpi .ru 


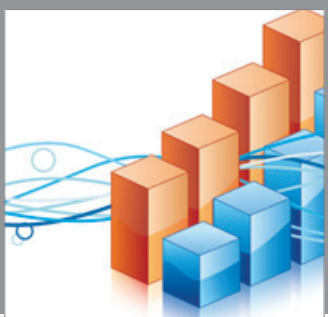

Advances in

Operations Research

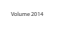

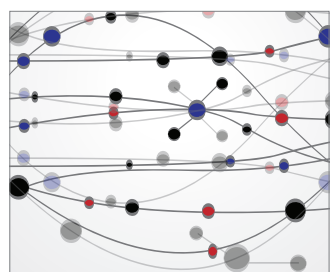

\section{The Scientific} World Journal
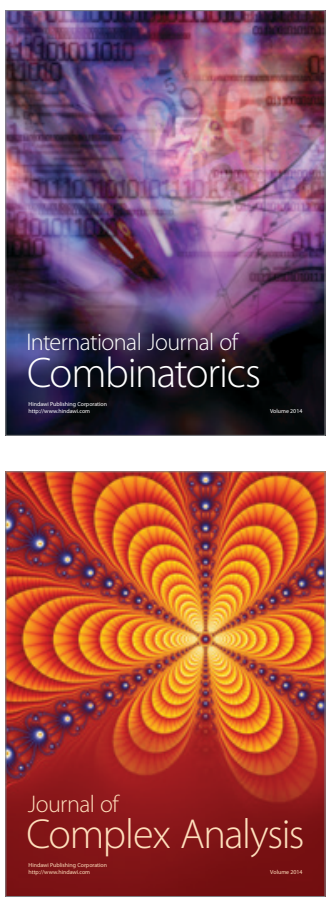

International Journal of

Mathematics and

Mathematical

Sciences
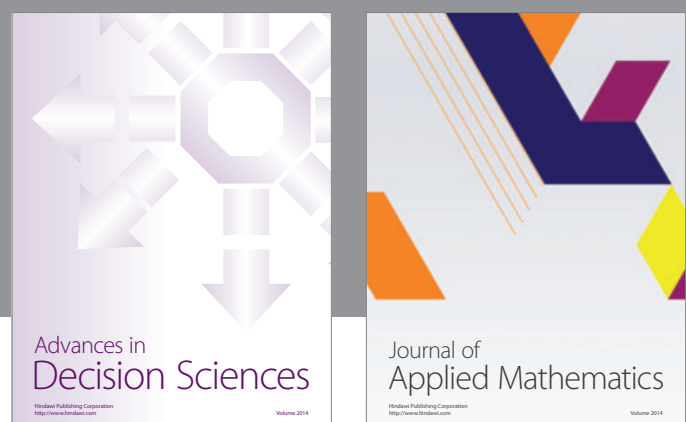

Journal of

Applied Mathematics
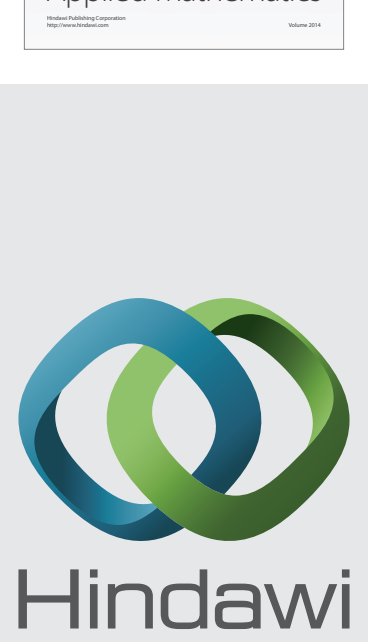

Submit your manuscripts at http://www.hindawi.com
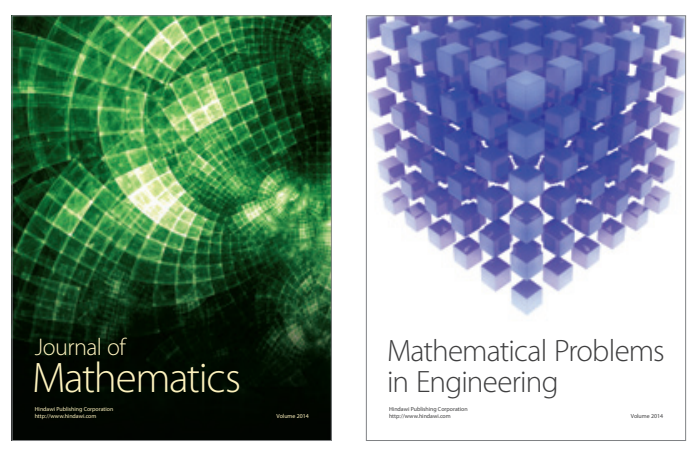

Mathematical Problems in Engineering
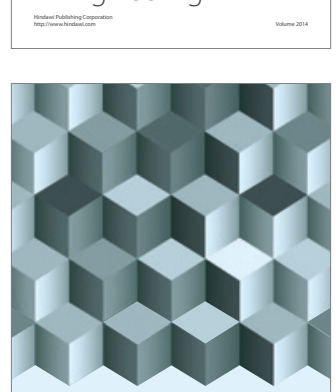

Journal of

Function Spaces
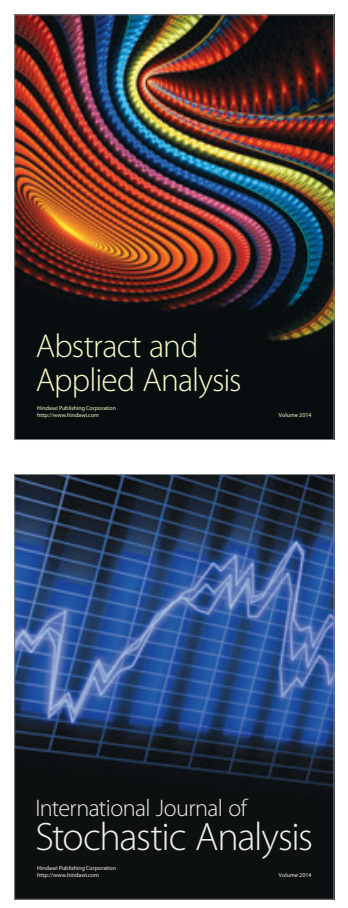

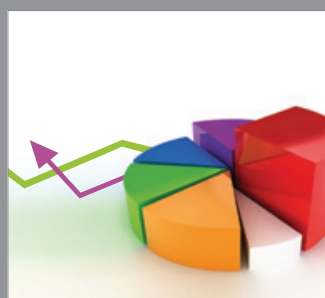

ournal of

Probability and Statistics

Promensencen
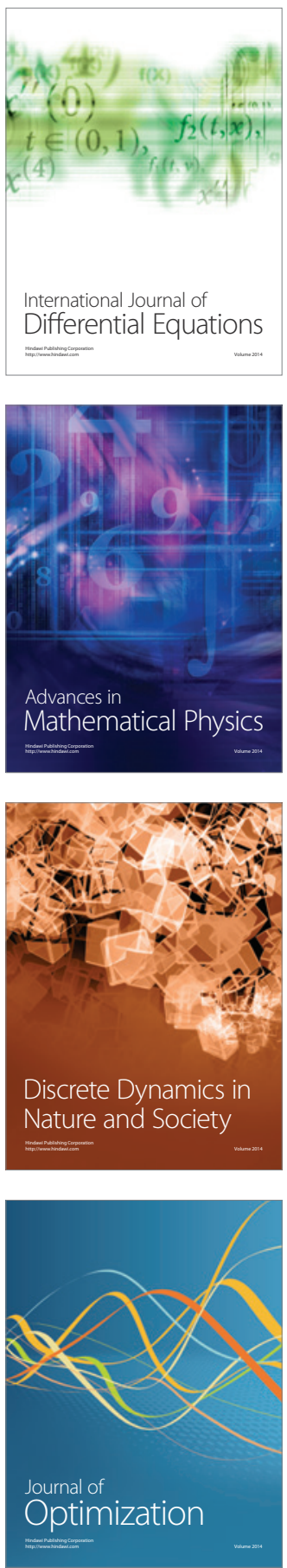